\title{
Interval-Valued Intuitionistic Fuzzy Multicriteria Group Decision Making Based on VIKOR and Choquet Integral
}

\author{
Chunqiao Tan and Xiaohong Chen \\ School of Business, Central South University, Changsha 410083, China \\ Correspondence should be addressed to Chunqiao Tan; chunqiaot@sina.com
}

Received 10 August 2013; Revised 2 October 2013; Accepted 17 October 2013

Academic Editor: Reinaldo Martinez Palhares

Copyright ( 92013 C. Tan and X. Chen. This is an open access article distributed under the Creative Commons Attribution License, which permits unrestricted use, distribution, and reproduction in any medium, provided the original work is properly cited.

\begin{abstract}
An effective decision making approach based on VIKOR and Choquet integral is developed to solve multicriteria group decision making problem with conflicting criteria and interdependent subjective preference of decision makers in a fuzzy environment where preferences of decision makers with respect to criteria are represented by interval-valued intuitionistic fuzzy sets. First, an intervalvalued intuitionistic fuzzy Choquet integral operator is given. Some of its properties are investigated in detail. The extended VIKOR decision procedure based on the proposed operator is developed for solving the multicriteria group decision making problem where the interactive criteria weight is measured by Shapley value. An illustrative example is given for demonstrating the applicability of the proposed decision procedure for solving the multi-criteria group decision making problem in interval-valued intuitionistic fuzzy environment.
\end{abstract}

\section{Introduction}

The increasing complexity of the socioeconomic environments makes it less and less possible for a single decision maker to consider all relevant aspects of a problem. Hence, in order to get a more reasonable decision result, a decision organization, such as the board of directors of a company, which contains a collection of decision makers, is set up explicitly or implicitly to assess the alternatives. The analysis must be extended to account, somehow, for group decision makers, each one potentially exhibiting a unique preference structure, perceiving different consequences, and responding to a diverse array of aspirations [1].

Multiple criteria or attribute decision making (MCDM or MADM) problems is to find the best compromise solution among all feasible alternatives assessed on the basis of multiple criteria or attributes, both quantitative and qualitative. Due to the complex structure of the problem and conflicting nature of the criteria for multi-criteria decision making, trade-offs assessment is one of the most difficult issues in multi-criteria decision making. There may be no solution satisfying all criteria simultaneously. Thus, the solution is a set of noninferior solutions or a compromise solution according to the decision maker's preferences. By using compromise programming, the compromise solution was established by $\mathrm{Yu}$ [2] and Zeleny [3] for a problem with conflicting criteria and it can be helping the decision makers to reach a final solution. The compromise solution is a feasible solution, which is the closest to the ideal, and compromise means an agreement established by mutual concessions. Based on the compromise programming [3], many MCDM ranking methods have been investigated, such as the TOPSIS (technique for order preference by similarity to an ideal solution) method [4], the VIKOR (vlsekriterijumska optimizacija i kompromisno resenje in Serbian, meaning multicriteria optimization and compromise solution) method [5], the PROMETHEE (preference ranking organization method for enrichment evaluations) method [6], and the ELECTRE (elimination et choice translating reality) method [7].

As one of the compromise ranking approaches for multiple criteria decision making problems, The VIKOR method focuses on ranking and selecting from the alternatives with conflicting and different units criteria. The vIKOR method of compromise ranking determines a compromise solution that provides the maximum group utility for the majority and a minimum of individual regret [8] for the opponent. Within the VIKOR method, the compromise ranking could be performed by comparing the measure of closeness to the ideal 
alternative through the process of ranking and selecting a set of alternatives in the presence of conflicting criteria. The obtained compromise solution could be accepted by the decision makers because it provides a maximum group utility of the majority and a minimum individual regret of the opponent. The compromise solutions could be the base for negotiation, involving the decision makers' preference by criteria weights and a balance between total and individual satisfaction. The VIKOR has been developed for multi-criteria optimization in complex systems and enjoys a wide acceptance. Opricovic and Tzeng [9] made a comparative analysis of VIKOR and TOPSIS. Furthermore, Opricovic and Tzeng [10] extended the VIKOR method with a stability analysis determining the weight stability intervals and with tradeoffs analysis and also compared it deeply with the TOPSIS method, the PROMETHEE method, and the ELECTRE method. According to the comparisons, it is obvious that the VIKOR method has many advantages in handling the MCDM problems especially when they are with conflicting and noncommensurable criteria. Tzeng et al. [11] used and compared the VIKOR and TOPSIS methods in solving a public transportation problem. Sayadi et al. [12] extended the VIKOR method to MADM problem with interval numbers. Chang and Hsu [13] showed that the VIKOR method is advantageous for evaluating the relative environmental vulnerability of subdivisions in a watershed. Up to now, the VIKOR method has been applied widely in many fields, such as mountain destination choosing [9], alternative hydropower systems evaluating [9], forestation and forest preservation [14], postearthquake sustainable reconstruction [15], airlines service quality evaluation [16], and selection of the renewable energy project [17].

In complex decision making problems, imprecision and uncertainty are often involved in decision making process due to incomplete information, abundant information, conflicting evidence, ambiguous information, and subjective information [18-20]. The preference information provided by decision maker is also imprecise or uncertain due to time pressure, lack of data, or the decision maker's limited attention and information processing capacities. To adequately model such decision making situations, fuzzy set [21] and its higher order extensions such as intuitionistic fuzzy set (IFS) [22] and interval-valued intuitionistic fuzzy set (IVIFS) [23] have been successfully used to handle imprecise and uncertain human decision behaviors [20, 24, 25].

Recently, The VIKOR method has been developed for fuzzy multi-criteria decision making in complex systems. Opricovic and Tzeng [26] suggested using fuzzy logic for the VIKOR method. Büyüközkan and Ruan [27] extended the VIKOR method to effectively solve software evaluation problem under a fuzzy environment. Sanayei et al. [28] proposed a hierarchy MCDM model based on fuzzy sets theory and VIKOR method to deal with the supplier selection problems in the supply chain system. Opricovic [29] proposed fuzzy VIKOR method for water resources planning management. Chen and Wang [30] proposed a more efficient delivery approach for evaluating and assessing possible suppliers/vendors by using the fuzzy VIKOR method. Du and Liu [31] developed three extensions of the VIKOR method based on the expected values of the intuitionistic trapezoidal fuzzy numbers and the distances between the intuitionistic trapezoidal fuzzy numbers and the interval numbers, respectively. Devi [32] extended VIKOR method to an intuitionistic fuzzy environment for robot selection. Park et al. [33] extended the VIKOR method for dynamic intuitionistic fuzzy multiple attribute decision making. Park et al. [34] extended the concept of the VIKOR method to develop a methodology for solving the MCDM problems with intervalvalued intuitionistic fuzzy numbers.

It is worthwhile noting that the above different kinds of extensions of the VIKOR are based on the assumption that the criteria and subjective preferences of the decision maker are independent with the use of a linear-based mathematical model for facilitating the human decision making process, which is characterized by an independence axiom $[35,36]$. However, for real MCDM problems, there is always some degree of interdependent characteristics among criteria [3741]. For example, we are to evaluate a set of students in relation to three subjects: \{mathematics, physics, literature\}; we want to give more importance to science-related subjects than to literature, but on the other hand we want to give some advantage to students that are good both in literature and in any of the science-related subjects [38]. Individual decision makers may come from the same or similar research fields in a given situation. They often have similar knowledge, social status, and preference. As a result, their subjective preferences in the group decision-making process often show some interactive characteristics. Traditional additive aggregation operators such as the weighted average or ordered weighted averaging (OWA) operator [42] are based on an implicit assumption that the criteria or the preferences of individual decision maker is independent of each another. This leads to a wide application of the additive expected utility mode [35] based on the mutual preferential independence [36] for solving the multi-criteria group decision making problem. There are, however, some experiments that show that the additive expected utility model and the assumption of mutual preferential independence are often violated [43], which restricts the potential applications of the traditional additive aggregation operators to more extensive areas.

As the generalization of the weighted average operator and the OWA operator, the Choquet integral with respect to fuzzy measure [41] is an effective tool for extending the additive expected utility model that allow weakening the inherent independence hypotheses for modeling the subjective human decision making process [44]. In order to overcome the drawback of those extensions of the VIKOR, it is necessary to develop a new extension of the VIKOR method for fuzzy group multi-criteria decision making. The aim of this paper is to extend the classical VIKOR method to solve the fuzzy multi-criteria group decision making problems in interval-valued intuitionistic fuzzy environment where all the preference information provided by the decision maker is presented as interval-valued intuitionistic fuzzy set, where inter-dependent or interactive characteristics among criteria and preference of decision makers are also taken into account. The motivation and main features of the proposed extended VIKOR method in this paper are summarized as follows. 
(i) Although [34] extended the VIKOR for the multicriteria group decision making problems in interval-valued intuitionistic fuzzy environment, they did not take the interactive characteristics among preference of decision makers into account. An additive interval-valued intuitionistic fuzzy aggregation operator, the interval-valued intuitionistic fuzzy weighted geometric operator, was used to aggregate decision makers' preferences. This paper considers the interactive characteristics among decision makers' preferences, a nonlinear aggregation operator; interval-valued intuitionistic fuzzy Choquet integral operator, is proposed for aggregating decision makers' preferences where the interactive characteristics among decision-makers' preferences are expressed by means of fuzzy measure. It is shown that when the fuzzy measure is an additive fuzzy measure, decision-makers' subjective preferences are independent; the nonlinear aggregation operator is reduced to an additive interval-valued intuitionistic fuzzy aggregation operator.

(ii) When inter-dependent or interactive characteristics among criteria are taken into account, the weights of interactive criteria should not be determined by traditional methods. In this paper, we use fuzzy measure to measure the interdependent characteristics among criteria; then Shapley value is used to determine the interactive criteria weights, which reflects decision-maker's subjective preference to criteria as well as the inherent objective characteristics of criteria. It is shown that when the fuzzy measure is an additive fuzzy measure, the Shapley weights is reduced to the classical weights.

(iii) In the classical VIKOR, the criteria weights and decision-maker's weights are also given in advance. Furthermore, the classical VIKOR is only appropriate for the decision making problems with the crisp assessment information and cannot solve the fuzzy multi-criteria group decision making with interval-valued intuitionistic fuzzy sets. The fundamental characteristic of the interval-valued intuitionistic fuzzy set is that the value of the membership function and nonmembership function of the interval-valued intuitionistic fuzzy set is interval rather than exact number in $[0,1]$, respectively. The interval-valued intuitionistic fuzzy set is more suitable for dealing with imprecise and uncertain information than fuzzy set or intuitionistic fuzzy set. The extended VIKOR method has more flexible for dealing with fuzzy information.

(iv) Compared with the similar methods, the proposed method in this paper can maximize the group utility and minimize the individual regret, and the inter-dependent or interactive characteristics among criteria and preference of decision makers are taken into account simultaneously, which make the decision result more reasonable. The decisionmakers can adjust the coefficient of decision mechanism to reflect the different importance of group utility and individual regret, which greatly enhances the flexibility of the proposed method. Moreover, though the proposed method is developed to solve multi-criteria group decision making with interval-valued intuitionistic fuzzy set, it also can be used to solve MCDM with intuitionistic fuzzy set.

The remaining of this paper is organized as follows. We first present some preliminary concepts including fuzzy measure, Choquet integral, and interval-valued intuitionistic fuzzy set in Sections 2 and 3, respectively. An intervalvalued intuitionistic fuzzy Choquet integral operator is given, and some of its properties are investigated in detail in Section 4. In Section 5, subjective and objective weights of criteria are proposed according to fuzzy measure and Shapley value. In Section 6, the extended VIKOR decision procedure based on the proposed operator is developed for effectively tackling the multi-criteria group decision making problem in an interval-valued intuitionistic fuzzy environment. In Section 7, we give an example for illustrating the applicability of the proposed decision procedure for solving the real world decision problem. Finally, the paper is concluded with some observations in Section 8.

\section{Fuzzy Measure and Choquet Integral}

Fuzzy measure (a nonadditive measure) is proposed for addressing the issue of nonadditivity in the traditional additive utility model in decision making. It can be defined as follows [41].

Definition 1. Let $X=\left\{x_{1}, x_{2}, \ldots, x_{n}\right\}$ be a universe of discourse and let $P(X)$ be the power set of $X$. A fuzzy measure on $X$ is a set function $\mu: P(X) \rightarrow[0,1]$, satisfying the following conditions:

(i) $\mu(\emptyset)=0, \mu(X)=1$.

(ii) If $A, B \in P(X)$ and $A \subseteq B$, then $\mu(A) \leq \mu(B)$.

In the multi-criteria decision making, $\mu(A)$ can be viewed as the grade of subjective importance of decision criteria set $A$. Thus, in addition to the usual weights on criteria taken separately, weights on any combination of criteria are also defined. To determine such fuzzy measures, it needs to find $2^{n}-2$ values for $n$ criteria in a given multi-criteria group decision making situation. With the definition as above, the values of $\mu(\emptyset)$ and $\mu(X)$ are always equal to 0 and 1 , respectively. It is obvious that such an evaluation process is quite complex with the structure being difficult to grasp. To reduce the computational complexity, the $\lambda$-fuzzy measure $g$ that acts as a special kind of fuzzy measure is proposed which is defined as follows [45].

Definition 2. Let $X=\left\{x_{1}, x_{2}, \ldots, x_{n}\right\}$ be a universe of discourse; a fuzzy measure $g$ on $X$ is called $\lambda$-fuzzy measure if it satisfies the following conditions:

$$
g(A \cup B)=g(A)+g(B)+\lambda g(A) g(B),
$$

where $\lambda \in(-1, \infty)$ for all $A, B \in P(X)$ and $A \cap B=\emptyset$.

When $\lambda=0, g(A \cup B)=g(A)+g(B)$. This means that $A$ and $B$ are mutually independent, that is; $g$ is additive measure and there exists no interaction between $A$ and $B$. $\lambda \neq 0$ indicates that $g$ is nonadditive and there exist some degrees of interdependency between $A$ and $B$. If $\lambda>0$, $g(A \cup B)>g(A)+g(B)$. This implies that the set $\{A, B\}$ has multiplicative effect. If $\lambda<0, g(A \cup B)<g(A)+g(B)$. This shows that the set $\{A, B\}$ has substitutive effect. With the use 
of parameter $\lambda$, the interaction between criteria in multicriteria decision making can be adequately represented.

If $X$ is a finite set, $\bigcup_{i=1}^{n} x_{i}=X$. The $\lambda$-fuzzy measure $g$ satisfies the following equation:

$$
\begin{aligned}
g(X) & =g\left(\bigcup_{i=1}^{n} x_{i}\right) \\
& = \begin{cases}\frac{1}{\lambda}\left(\prod_{i=1}^{n}\left[1+\lambda g\left(x_{i}\right)\right]-1\right), & \text { if } \lambda \neq 0, \\
\sum_{i=1}^{n} g\left(x_{i}\right), & \text { if } \lambda=0,\end{cases}
\end{aligned}
$$

where $x_{i} \cap x_{j}=\emptyset$ for all $i, j=1,2, \ldots, n$ and $i \neq j . g\left(x_{i}\right)$ for a subset with a single element $x_{i}$ is called a fuzzy density, denoted by $g_{i}=g\left(x_{i}\right)$.

Especially for every subset $A \in P(X)$, we have

$$
g(A)= \begin{cases}\frac{1}{\lambda}\left(\prod_{i \in A}[1+\lambda g(i)]-1\right), & \text { if } \lambda \neq 0, \\ \sum_{i \in A} g(i), & \text { if } \lambda=0 .\end{cases}
$$

The value $\lambda$ can be uniquely determined based on (2) from $g(X)=1$ by solving the equation

$$
\lambda+1=\prod_{i=1}^{n}\left(1+\lambda g_{i}\right)
$$

It should be noted that $\lambda$ can also be uniquely determined by $g(X)=1$.

As a generalization of the linear Lebesgue integral, the Choquet integral is defined as follows [41].

Definition 3. Let $f$ be a positive real-valued function on $X$, and let $\mu$ be a fuzzy measure on $X$. The discrete Choquet integral of $f$ with respect to $\mu$ is defined by

$$
C_{\mu}(f)=\sum_{i=1}^{n} f\left(x_{(i)}\right)\left[\mu\left(A_{(i)}\right)-\mu\left(A_{(i+1)}\right)\right],
$$

where the subscript $(\cdot)$ indicates a permutation on $X$ such that $f\left(x_{(1)}\right) \leq f\left(x_{(2)}\right) \leq \cdots \leq f\left(x_{(n)}\right)$. Also, $A_{(i)}=\left\{x_{(i)}, \ldots, x_{(n)}\right\}$, and $A_{(n+1)}=\emptyset$.

The Choquet integral is popular in tackling real decision making problems as it coincides with the Lebesgue integral in which the measure is additive. An additive measure may be directly tied to the notions of the additive expected utility theory and mutual preferential independence [36]. The Choquet integral is able to aggregate the criteria weighting information even when the mutual preferential independence is violated [44].

\section{Interval-Valued Intuitionistic Fuzzy Set}

Let $X$ be a universe of discourse; a fuzzy set $A$ in $X$ can be expressed as

$$
A=\left\{\left\langle x, t_{A}(x)\right\rangle \mid x \in X\right\}
$$

where $t_{A}: X \rightarrow[0,1]$ is a membership function which characterizes the degree of membership of the element $x$ in the set $A$. The main characteristic of fuzzy sets is that a membership function value is assigned to each element $x$ in a universe of discourse $X$. A nonmembership degree equals to one minus the membership degree [21]. This means that this single membership degree combines the evidence for $x$ and the evidence against $x$ without indicating how much there is for each. This single membership value therefore tells us nothing about the lack of knowledge in describing the element $x$. In real applications, however, the information about an object corresponding to a fuzzy concept may be incomplete. That means that the sum of the membership degree and the nonmembership degree of that object in a universe of discourse corresponding to a fuzzy concept may be less than one. In the fuzzy sets theory, there is no means to incorporate the lack of knowledge with the membership degree. To effectively deal with this issue, intuitionistic fuzzy set [22] is introduced as an extension of the traditional fuzzy set as follows.

Definition 4. Let $X=\left\{x_{1}, x_{2}, \ldots, x_{n}\right\}$ be a universe of discourse; an intuitionistic fuzzy set in $X$ is an expression $A$ given by

$$
A=\left\{\left\langle x, t_{A}(x), f_{A}(x)\right\rangle \mid x \in X\right\}
$$

where $t_{A}: X \rightarrow[0,1], f_{A}: X \rightarrow[0,1]$ with the condition: $0 \leq t_{A}(x)+f_{A}(x) \leq 1$, for all $x$ in $X$. The numbers $t_{A}(x)$ and $f_{A}(x)$ represent the degree of membership and the degree of nonmembership of the element $x$ in the set $A$, respectively.

For each intuitionistic fuzzy set $A$ in $X$, if $\pi_{A}(x)=1-$ $t_{A}(x)-f_{A}(x)$, for all $x \in X$, then $\pi_{A}(x)$ is called the degree of indeterminacy of $x$ to $A$. Especially, if $\pi_{A}(x)=1-t_{A}(x)-$ $f_{A}(x)=0$, for all $x \in X$, the intuitionistic fuzzy set $A$ is reduced to a fuzzy set.

Interval-valued intuitionistic fuzzy set [23] is a generalization of the intuitionistic fuzzy sets. The fundamental characteristic of the interval-valued intuitionistic fuzzy set is that the value of the membership function and nonmembership function of the interval-valued intuitionistic fuzzy set is interval rather than exact number.

Definition 5. Let $X$ be a universe of discourse and let $D[0,1]$ be the set of all closed subintervals of the interval $[0,1]$. An interval-valued intuitionistic fuzzy set $A$ in $X$ is an expression given by

$$
A=\left\{\left\langle x, t_{A}(x), f_{A}(x)\right\rangle \mid x \in X\right\}
$$

where $t_{A}: X \rightarrow D[0,1], f_{A}: X \rightarrow D[0,1]$ with the condition $0 \leq \sup t_{A}(x)+\sup f_{A}(x) \leq 1$. The intervals $t_{A}(x)$ and $f_{A}(x)$ denote, respectively, the degree of belongingness and the degree of nonbelongingness of the element $x$ to the set $A$.

For any two intervals $[a, b]$ and $[c, d]$ with $b+d \leq 1$ belonging to $D[0,1]$, let $t_{A}(x)=[a, b]$, and $f_{A}(x)=[c, d]$. An interval-valued intuitionistic fuzzy set can be denoted by 
$A=\{\langle x,[a, b],[c, d]\rangle \mid x \in X\}$. In this paper, $([a, b],[c, d])$ is referred as an interval-valued intuitionistic fuzzy value. For convenience, let $\Omega$ be the set of all intervalvalued intuitionistic fuzzy values on $X$. Obviously, $\widetilde{a}^{+}=([1,1],[0,0])$ and $\tilde{a}=([0,0],[1,1])$ are the largest and smallest interval-valued intuitionistic fuzzy values, respectively. A distance measure between inter-valued intuitionistic fuzzy values is defined as follows [24].

Definition 6. Let $X=\left\{x_{1}, \ldots, x_{n}\right\}$ be a universe of discourse, and $\widetilde{a}=\left(\left[a_{i}, b_{i}\right],\left[c_{i}, d_{i}\right]\right)$ and $\widetilde{b}=\left(\left[a_{i}^{\prime}, b_{i}^{\prime}\right],\left[c_{i}^{\prime}, d_{i}^{\prime}\right]\right)(i=$ $1, \ldots, n)$ be two interval-valued intuitionistic fuzzy values on $X$;

$$
d(\tilde{a}, \widetilde{b})=\frac{1}{4} \sum_{i=1}^{n}\left|a_{i}-a_{i}^{\prime}\right|+\left|b_{i}-b_{i}^{\prime}\right|+\left|c_{i}-c_{i}^{\prime}\right|+\left|d_{i}-d_{i}^{\prime}\right|
$$

is called the normalized Hamming distance between $\widetilde{a}$ and $\widetilde{b}$, if

$$
\begin{aligned}
& d(\tilde{a}, \widetilde{b})=\frac{1}{4} \sum_{i=1}^{n} w_{i}\left(\left|a_{i}-a_{i}^{\prime}\right|+\left|b_{i}-b_{i}^{\prime}\right|\right. \\
& \left.+\left|c_{i}-c_{i}^{\prime}\right|+\left|d_{i}-d_{i}^{\prime}\right|\right),
\end{aligned}
$$

where $w=\left(w_{1}, w_{2}, \ldots, w_{n}\right)$ is the weight vector of $x_{j}$ such that $w_{i} \in[0,1]$ and $\sum_{i=1}^{n} w_{i}=1$, and $d(\widetilde{a}, \widetilde{b})$ is called the weighted Hamming distance between $\widetilde{a}$ and $\widetilde{b}$.

The following expressions are defined for any two interval-valued intuitionistic fuzzy values $\widetilde{a}_{1}=\left(\left[a_{1}, b_{1}\right],\left[c_{1}, d_{1}\right]\right)$ and $\widetilde{a}_{2}=\left(\left[a_{2}, b_{2}\right],\left[c_{2}, d_{2}\right]\right)[24]$ :

(i)

$$
\tilde{a}_{1} \leq \tilde{a}_{2} \quad \text { iff } b_{1} \leq b_{2}, a_{1} \leq a_{2}, d_{1} \geq d_{2}, c_{1} \geq c_{2},
$$

(ii)

$$
\widetilde{a}_{1}=\widetilde{a}_{2} \text { iff } b_{1}=b_{2}, a_{1}=a_{2}, d_{1}=d_{2}, c_{1}=c_{2} .
$$

Equation (11), however, is not always satisfied in some decision making situations. To address this issue, the following order relation between interval-valued intuitionistic fuzzy values is defined [46].

Definition 7. Let $\widetilde{a}_{1}=\left(\left[a_{1}, b_{1}\right],\left[c_{1}, d_{1}\right]\right)$ and $\widetilde{a}_{2}=\left(\left[a_{2}, b_{2}\right]\right.$, $\left.\left[c_{2}, d_{2}\right]\right)$ be two interval-valued intuitionistic fuzzy values, let $S\left(\widetilde{a}_{1}\right)=\left(a_{1}-c_{1}+b_{1}-d_{1}\right) / 2$ and $S\left(\widetilde{a}_{2}=\left(a_{2}-c_{2}+b_{2}-d_{2}\right) / 2\right.$ be the score functions of $\widetilde{a}_{1}$ and $\widetilde{a}_{2}$, respectively, and let $H\left(\widetilde{a}_{1}\right)=$ $\left(a_{1}+c_{1}+b_{1}+d_{1}\right) / 2$ and $H\left(\widetilde{a}_{2}\right)=\left(a_{2}+c_{2}+b_{2}+d_{2}\right) / 2$ be the accuracy functions of $\widetilde{a}_{1}$ and $\widetilde{a}_{2}$, respectively:

(a) if $S\left(\widetilde{a}_{1}\right)<S\left(\widetilde{a}_{2}\right), \widetilde{a}_{1}$ is smaller than $\widetilde{a}_{2}$, denoted by $\widetilde{a}_{1}<$ $\tilde{a}_{2}$

(b) if $S\left(\widetilde{a}_{1}\right)=S\left(\widetilde{a}_{2}\right)$ :

(i) if $H\left(\widetilde{a}_{1}\right)<H\left(\widetilde{a}_{2}\right), \widetilde{a}_{1}$ is smaller than $\widetilde{a}_{2}$, denoted by $\widetilde{a}_{1}<\widetilde{a}_{2}$;

(ii) if $H\left(\widetilde{a}_{1}\right)=H\left(\widetilde{a}_{2}\right), \widetilde{a}_{1}$ and $\widetilde{a}_{2}$ represent the same information, denoted by $\tilde{a}_{1}=\widetilde{a}_{2}$.

\section{Interval-Valued Intuitionistic Fuzzy Choquet Integral Operator}

In interval-valued intuitionistic fuzzy multi-criteria group decision making problems, some additive linear operators, such as interval-valued intuitionistic fuzzy weighted averaging [46], interval-valued intuitionistic fuzzy weighted geometric $[47,48]$, or interval-valued intuitionistic fuzzy OWA [48] operators, are applied for aggregating the decision maker's preference information. Existing research for multicriteria group decision making does not adequately address the issue of interdependencies among criteria or decisionmakers' preference. Inspired by the idea of the Choquet integral operator in decision making, in this section, an intervalvalued intuitionistic fuzzy Choquet integral operator is given for effectively addressing the issue of interdependence among decision-makers' preference in a fuzzy environment [49, 50]. And some of its properties are investigated in detail. The relation between this operator and the additive linear interval-valued intuitionistic fuzzy aggregation operators is analyzed. To do this, first two operational laws for intervalvalued intuitionistic fuzzy values $[24,46]$ are introduced.

Definition 8. Let $\widetilde{a}_{1}=\left(\left[a_{1}, b_{1}\right],\left[c_{1}, d_{1}\right]\right)$ and $\widetilde{a}_{2}=\left(\left[a_{2}, b_{2}\right]\right.$, $\left.\left[c_{2}, d_{2}\right]\right)$ be two interval-valued intuitionistic fuzzy values;

(i) $\widetilde{a}_{1}+\widetilde{a}_{2}=\left(\left[a_{1}+a_{2}-a_{1} a_{2}, b_{1}+b_{2}-b_{1} b_{2}\right],\left[c_{1} c_{2}, d_{1} d_{2}\right]\right)$;

(ii) $\lambda \widetilde{a}_{1}=\left(\left[1-\left(1-a_{1}\right)^{\lambda}, 1-\left(1-b_{1}\right)^{\lambda}\right],\left[c_{1}^{\lambda}, d_{1}^{\lambda}\right]\right), \lambda>0$.

These two operational laws have two properties described as follows.

Proposition 9. Let $\widetilde{a}_{1}=\left(\left[a_{1}, b_{1}\right],\left[c_{1}, d_{1}\right]\right)$ and $\widetilde{a}_{2}=\left(\left[a_{2}, b_{2}\right]\right.$, $\left.\left[c_{2}, d_{2}\right]\right)$ be two interval-valued intuitionistic fuzzy values, and let $\widetilde{c}=\widetilde{a}_{1}+\widetilde{a}_{2}$ and $\widetilde{d}=\lambda \widetilde{a}_{1}$; both $\widetilde{c}$ and $\widetilde{d}$ are also intervalvalued intuitionistic fuzzy values.

Proposition 10. Let $\widetilde{a}_{1}=\left(\left[a_{1}, b_{1}\right],\left[c_{1}, d_{1}\right]\right)$ and $\widetilde{a}_{2}=\left(\left[a_{2}, b_{2}\right]\right.$, $\left.\left[c_{2}, d_{2}\right]\right)$ be two interval-valued intuitionistic fuzzy values, for all $\lambda_{1}, \lambda_{2}>0$,

(i) $\widetilde{a}_{1}+\widetilde{a}_{2}=\widetilde{a}_{2}+\widetilde{a}_{1}$;

(ii) $\lambda_{1}\left(\widetilde{a}_{1}+\widetilde{a}_{2}\right)=\lambda_{1} \widetilde{a}_{1}+\lambda_{1} \widetilde{a}_{2}$;

(iii) $\lambda_{1} \widetilde{a}_{1}+\lambda_{2} \widetilde{a}_{1}=\left(\lambda_{1}+\lambda_{2}\right) \widetilde{a}_{1}$.

According to these operational laws, an interval-valued intuitionistic fuzzy Choquet integral operator is defined as follows [49, 51].

Definition 11. Let $\widetilde{a}_{i}=\left(\left[a_{i}, b_{i}\right],\left[c_{i}, d_{i}\right]\right)(i=1,2, \ldots, n)$ be a collection of interval-valued intuitionistic fuzzy values on 
$X$, and let $\mu$ be a fuzzy measure on $X$. The discrete intervalvalued intuitionistic fuzzy Choquet integral (I-IFC) of $\widetilde{a}_{i}$ with respect to $\mu$ is defined by

$$
\begin{aligned}
\operatorname{I-IFC}_{\mu}\left(\tilde{a}_{1}, \ldots, \tilde{a}_{n}\right) \\
=\tilde{a}_{(1)}\left(\mu\left(A_{(1)}\right)-\mu\left(A_{(2)}\right)\right)+\tilde{a}_{(2)}\left(\mu\left(A_{(2)}\right)-\mu\left(A_{(3)}\right)\right) \\
\quad+\cdots+\tilde{a}_{(n)}\left(\mu\left(A_{(n)}\right)-\mu\left(A_{(n+1)}\right)\right) \\
=\sum_{i=1}^{n} \tilde{a}_{(i)}\left(\mu\left(A_{(i)}\right)-\mu\left(A_{(i+1)}\right)\right) .
\end{aligned}
$$

Further, the aggregated value is also an interval-valued intuitionistic fuzzy value, and

$$
\begin{gathered}
\operatorname{I-IFC}_{\mu}\left(\tilde{a}_{1}, \ldots, \tilde{a}_{n}\right) \\
=\left(\left[1-\prod_{i=1}^{n}\left(1-a_{(i)}\right)^{\mu\left(A_{(i)}\right)-\mu\left(A_{(i+1)}\right)},\right.\right. \\
\left.1-\prod_{i=1}^{n}\left(1-b_{(i)}\right)^{\mu\left(A_{(i)}\right)-\mu\left(A_{(i+1)}\right)}\right], \\
{\left[\prod_{i=1}^{n}\left(c_{(i)}\right)^{\mu\left(A_{(i)}\right)-\mu\left(A_{(i+1)}\right)},\right.} \\
\left.\left.\prod_{i=1}^{n}\left(d_{(i)}\right)^{\mu\left(A_{(i)}\right)-\mu\left(A_{(i+1)}\right)}\right]\right),
\end{gathered}
$$

where the subscript $(\cdot)$ indicates a permutation on $X$ such that $\tilde{a}_{(1)} \leq \tilde{a}_{(2)} \leq \cdots \leq \widetilde{a}_{(n)}$. And $A_{(i)}=((i), \ldots,(n)), A_{(n+1)}=\emptyset$.

Remark 12. Obviously if interval-valued intuitionistic fuzzy value $\tilde{a}_{i}=\left(\left[a_{i}, b_{i}\right],\left[c_{i}, d_{i}\right]\right)(i=1,2, \ldots, n)$ is reduced to intuitionistic fuzzy values, the interval-valued intuitionistic fuzzy Choquet integral of $\tilde{a}_{i}$ with respective to $\mu$ is reduced to an intuitionistic fuzzy Choquet integral operator [52].

Remark 13. Let $\widetilde{a}_{i}=\left(\left[a_{i}, b_{i}\right],\left[c_{i}, d_{i}\right]\right)$ and $\widetilde{b}_{i}=\left(\left[a_{i}^{\prime}, b_{i}^{\prime}\right]\right.$, $\left.\left[c_{i}^{\prime}, d_{i}^{\prime}\right]\right)(i=1,2, \ldots, n)$ be two collections of intervalvalued intuitionistic fuzzy values on $X$. Since $a_{i}, b_{i}, c_{i}, d_{i}$, $a_{i}^{\prime}, b_{i}^{\prime}, c_{i}^{\prime}, d_{i}^{\prime} \in[0,1]$ for any $i$, if we assume that $T_{P}\left(c_{i}, c_{i}^{\prime}\right)=c_{i} c_{i}^{\prime}$, $T_{P}\left(d_{i}, d_{i}^{\prime}\right)=d_{i} d_{i}^{\prime}, S_{P}\left(a_{i}, a_{i}^{\prime}\right)=a_{i}+a_{i}^{\prime}-a_{i} a_{i}^{\prime}$, and $S_{P}\left(b_{i}, b_{i}^{\prime}\right)=$ $b_{i}+b_{i}^{\prime}-b_{i} b_{i}^{\prime}$, then $T_{P}\left(c_{i}, c_{i}^{\prime}\right)$ and $T_{P}\left(d_{i}, d_{i}^{\prime}\right)$ are two of the basic $t$-norms, called the product, which is satisfying the following properties [53]: $T_{P}(x, 1)=x$ (boundary); $T_{P}(x, y) \leq T_{P}(x, z)$ whenever $y \leq z$ (monotonicity); $T_{P}(x, y)=T_{P}(y, x)$ (commutativity); and $T_{P}\left(x, T_{P}(y, z)\right)=T_{P}\left(T_{P}(x, y)\right.$ ) (associativity), where $x, y, z \in[0,1] . S_{P}\left(a_{i}, a_{i}^{\prime}\right)$ and $S_{P}\left(b_{i}, b_{i}^{\prime}\right)$ are two of the basic $t$-conorms, called the probabilistic sum, and $S_{P}$ is also called the dual $t$-conorm of $T_{P}$, which is satisfying the boundary; that is, $S_{P}(x, 0)=x$, monotonicity, commutativity, and associativity [53]. The associativity of $t$ norms and $t$-conorms allows us to extend the product $T_{P}$ and probabilistic sum $S_{P}$ in unique way to an $n$-ary operation in the usual way by induction, defining for each $n$-tuple $\left(x_{1}, x_{2}, \ldots, x_{n}\right) \in[0,1]^{n}$ and $\left(y_{1}, y_{2}, \ldots, y_{n}\right) \in[0,1]^{n}$, respectively,

$$
\begin{aligned}
T_{P}\left(x_{1}, x_{2}, \ldots, x_{n}\right) & =T_{i=1}^{n} x_{i} \\
& =T_{P}\left(\stackrel{n}{T}_{i=1}^{n-1} x_{i}, x_{n}\right)=\prod_{i=1}^{n} x_{i}, \\
S_{P}\left(y_{1}, y_{2}, \ldots, y_{n}\right) & ={\underset{S}{P}}_{i=1}^{n} y_{i} \\
& =S_{P}\left({\stackrel{n}{S_{P}}}_{i=1}^{n-1} y_{i}, y_{n}\right)=1-\prod_{i=1}^{n}\left(1-y_{i}\right) .
\end{aligned}
$$

Assume that $y_{i}=1-\left(1-a_{(i)}\right)^{\mu\left(A_{(i)}\right)-\mu\left(A_{(i+1)}\right)}, y_{i}^{\prime}=1-$ $\left(1-b_{(i)}\right)^{\mu\left(A_{(i)}\right)-\mu\left(A_{(i+1)}\right)}$, and $x_{i}=\left(c_{(i)}\right)^{\mu\left(A_{(i)}\right)-\mu\left(A_{(i+1)}\right)}, x_{i}^{\prime}=$ $\left(d_{(i)}\right)^{\mu\left(A_{(i)}\right)-\mu\left(A_{(i+1)}\right)}$,

$$
\begin{aligned}
\operatorname{I-IFC}_{\mu}\left(\tilde{a}_{1}, \ldots, \tilde{a}_{n}\right) & \\
= & \left(\left[S_{P}\left(y_{1}, \ldots, y_{n}\right), S_{P}\left(y_{1}^{\prime}, \ldots, y_{n}^{\prime}\right)\right],\right. \\
& {\left.\left[T_{P}\left(x_{1}, \ldots, x_{n}\right), T_{P}\left(x_{1}^{\prime}, \ldots, x_{n}^{\prime}\right)\right]\right) . }
\end{aligned}
$$

This means that the interval-valued intuitionistic fuzzy Choquet integral operator can be represented by one of the basic $t$-norms $T_{P}$ and $t$-conorms $S_{P}$.

In the following, we discuss some desirable properties about the interval-valued intuitionistic fuzzy Choquet integral operator.

Proposition 14. Let $\tilde{a}_{i}=\left(\left[a_{i}, b_{i}\right],\left[c_{i}, d_{i}\right]\right)(i=1,2, \ldots, n)$ be a collection of interval-valued intuitionistic fuzzy values on $X$, and let $\mu$ be a fuzzy measure on $X$. If all $\widetilde{a}_{i}(i=1,2, \ldots, n)$ are equal; that is, for all $i, \tilde{a}_{i}=\tilde{a}=([a, b],[c, d]), I-I F C_{\mu}\left(\tilde{a}_{1}\right.$, $\left.\ldots, \tilde{a}_{n}\right)=\tilde{a}$.

Proof. According to (14), we have if for all $i(i=1,2, \ldots, n)$, $\widetilde{a}_{i}=\widetilde{a}$,

$$
\begin{gathered}
\operatorname{I-IFC}_{\mu}\left(\tilde{a}_{1}, \ldots, \widetilde{a}_{n}\right) \\
=\left(\left[1-(1-a)^{\sum_{i=1}^{n} \mu\left(A_{(i)}\right)-\mu\left(A_{(i+1)}\right)},\right.\right. \\
\left.1-(1-b)^{\sum_{i=1}^{n} \mu\left(A_{(i)}\right)-\mu\left(A_{(i+1)}\right)}\right], \\
{\left[c^{\sum_{i=1}^{n} \mu\left(A_{(i)}\right)-\mu\left(A_{(i+1)}\right)},\right.} \\
\left.\left.d^{\sum_{i=1}^{n} \mu\left(A_{(i)}\right)-\mu\left(A_{(i+1)}\right)}\right]\right) .
\end{gathered}
$$

Since $\sum_{i=1}^{n}\left(\mu\left(A_{(i)}\right)-\mu\left(A_{(i+1)}\right)\right)=1$, we have that

$$
\operatorname{I}_{-I F C}\left(\tilde{a}_{1}, \ldots, \tilde{a}_{n}\right)=([a, b],[c, d])=\tilde{a} .
$$


Journal of Applied Mathematics

7

Proposition 15. Let $\tilde{a}_{i}=\left(\left[a_{i}, b_{i}\right],\left[c_{i}, d_{i}\right]\right)$ and $\tilde{b}_{i}=\left(\left[a_{i}^{\prime}, b_{i}^{\prime}\right]\right.$, $\left.\left[c_{i}^{\prime}, d_{i}^{\prime}\right]\right)(i=1,2, \ldots, n)$ be two collections of interval -valued intuitionistic fuzzy values on $X$, and let $\mu$ be a fuzzy measure on $X$. The subscript $(\cdot)$ indicates a permutation such that $\tilde{a}_{(1)} \leq$ $\cdots \leq \tilde{a}_{(n)}$ and $\tilde{b}_{(1)} \leq \cdots \leq \tilde{b}_{(n)}$. If $b_{(i)} \leq b_{(i)}^{\prime}, a_{(i)} \leq a_{(i)}^{\prime}$ and $d_{(i)} \geq d_{(i)}^{\prime}, c_{(i)} \geq c_{(i)}^{\prime}$ for all I; that is, $\widetilde{a}_{(i)} \leq \widetilde{b}_{(i)}$,

$$
I-I F C_{\mu}\left(\widetilde{a}_{1}, \ldots, \widetilde{a}_{n}\right) \leq I-I F C_{\mu}\left(\widetilde{b}_{1}, \ldots, \widetilde{b}_{n}\right) .
$$

Proof. Since $A_{(i+1)} \subseteq A_{(i)}$, then $\mu\left(A_{(i)}\right)-\mu\left(A_{(i+1)}\right) \geq 0$. For all $i, b_{(i)} \leq b_{(i)}^{\prime}, a_{(i)} \leq a_{(i)}^{\prime}$ and $d_{(i)} \geq d_{(i)}^{\prime}, c_{(i)} \geq c_{(i)}^{\prime}$, $1-\prod_{i=1}^{n}\left(1-a_{(i)}\right)^{\mu\left(A_{(i)}\right)-\mu\left(A_{(i+1)}\right)} \leq 1-\prod_{i=1}^{n}\left(1-a_{(i)}^{\prime}\right)^{\mu\left(A_{(i)}\right)-\mu\left(A_{(i+1)}\right)}$, $1-\prod_{i=1}^{n}\left(1-b_{(i)}\right)^{\mu\left(A_{(i)}\right)-\mu\left(A_{(i+1)}\right)} \leq 1-\prod_{i=1}^{n}\left(1-b_{(i)}^{\prime}\right)^{\mu\left(A_{(i)}\right)-\mu\left(A_{(i+1)}\right)}$, $\prod_{i=1}^{n}\left(f_{\widetilde{a}_{(i)}}\right)^{\mu\left(A_{(i)}\right)-\mu\left(A_{(i+1)}\right)} \geq \quad \prod_{i=1}^{n}\left(f_{\widetilde{b}_{(i)}}\right)^{\mu\left(A_{(i)}\right)-\mu\left(A_{(i+1)}\right)}$, $\prod_{i=1}^{n}\left(d_{(i)}\right)^{\mu\left(A_{(i)}\right)-\mu\left(A_{(i+1)}\right)} \geq \prod_{i=1}^{n}\left(d_{(i)}^{\prime}\right)^{\mu\left(A_{(i)}\right)-\mu\left(A_{(i+1)}\right)}$.

According to (14) and (11), we have

$$
\mathrm{I}^{-\mathrm{IFC}_{\mu}}\left(\widetilde{a}_{1}, \ldots, \widetilde{a}_{n}\right) \leq \mathrm{I}_{-} \operatorname{IFC}_{\mu}\left(\widetilde{b}_{1}, \ldots, \widetilde{b}_{n}\right) .
$$

Proposition 16. Let $\widetilde{a}_{i}=\left(\left[a_{i}, b_{i}\right],\left[c_{i}, d_{i}\right]\right)(i=1,2, \ldots, n)$ be a collection of interval-valued intuitionistic fuzzy values on $X$, and let $\mu$ be a fuzzy measure on $X$. If

$$
\begin{aligned}
& \tilde{a}^{-}=\left(\left[\min _{i} a_{i}, \min _{i} b_{i}\right],\left[\max _{i} c_{i}, \max _{i} d_{i}\right]\right), \\
& \tilde{a}^{+}=\left(\left[\max _{i} a_{i}, \max _{i} b_{i}\right],\left[\min _{i} c_{i}, \min _{i} d_{i}\right]\right),
\end{aligned}
$$

then $\tilde{a}^{-} \leq I-I F C_{\mu}\left(\tilde{a}_{1}, \ldots, \tilde{a}_{n}\right) \leq \tilde{a}^{+}$.

Proof. For any $\tilde{a}_{i}=\left(\left[a_{i}, b_{i}\right],\left[c_{i}, d_{i}\right]\right)(i=1, \ldots, n)$, it is obvious that $\widetilde{a}$ and $\widetilde{a}^{+}$are interval-valued intuitionistic fuzzy values. Since $A_{(i+1)} \subseteq A_{(i)}$, then $\mu\left(A_{(i)}\right)-\mu\left(A_{(i+1)}\right) \geq 0$. Let the subscript $(\cdot)$ indicate a permutation such that $\widetilde{a}_{(1)} \leq \cdots \leq \widetilde{a}_{(n)}$; for all $i$, we have

$$
\begin{array}{ll}
\min _{i} a_{i} \leq a_{(i)} \leq \max _{i} a_{i}, & \min _{i} b_{i} \leq b_{(i)} \leq \max _{i} b_{i}, \\
\min _{i} c_{i} \leq c_{(i)} \leq \max _{i} c_{i}, & \min _{i} d_{i} \leq d_{(i)} \leq \max _{i} d_{i} .
\end{array}
$$

Thus,

$$
\begin{aligned}
& 1-\prod_{i=1}^{n}\left(1-\min _{i} a_{i}\right)^{\mu\left(A_{(i)}\right)-\mu\left(A_{(i+1)}\right)} \\
& \quad \leq 1-\prod_{i=1}^{n}\left(1-a_{(i)}\right)^{\mu\left(A_{(i)}\right)-\mu\left(A_{(i+1)}\right)} \\
& \quad \leq 1-\prod_{i=1}^{n}\left(1-\max _{i} a_{i}\right)^{\mu\left(A_{(i)}\right)-\mu\left(A_{(i+1)}\right)},
\end{aligned}
$$

$$
\begin{aligned}
& 1-\prod_{i=1}^{n}\left(1-\min _{i} b_{i}\right)^{\mu\left(A_{(i)}\right)-\mu\left(A_{(i+1)}\right)} \\
& \leq 1-\prod_{i=1}^{n}\left(1-b_{(i)}\right)^{\mu\left(A_{(i)}\right)-\mu\left(A_{(i+1)}\right)} \\
& \leq 1-\prod_{i=1}^{n}\left(1-\max _{i} b_{i}\right)^{\mu\left(A_{(i)}\right)-\mu\left(A_{(i+1)}\right)}, \\
& \prod_{i=1}^{n}\left(\min _{i} c_{i}\right)^{\mu\left(A_{(i)}\right)-\mu\left(A_{(i+1)}\right)} \\
& \leq \prod_{i=1}^{n}\left(c_{(i)}\right)^{\mu\left(A_{(i)}\right)-\mu\left(A_{(i+1)}\right)} \\
& \leq \prod_{i=1}^{n}\left(\max _{i} c_{i}\right)^{\mu\left(A_{(i)}\right)-\mu\left(A_{(i+1)}\right)},
\end{aligned}
$$

$$
\begin{aligned}
& \prod_{i=1}^{n}\left(\min _{i} d_{i}\right)^{\mu\left(A_{(i)}\right)-\mu\left(A_{(i+1)}\right)} \\
& \leq \prod_{i=1}^{n}\left(d_{(i)}\right)^{\mu\left(A_{(i)}\right)-\mu\left(A_{(i+1)}\right)} \\
& \leq \prod_{i=1}^{n}\left(\max _{i} d_{i}\right)^{\mu\left(A_{(i)}\right)-\mu\left(A_{(i+1)}\right)} ;
\end{aligned}
$$

$$
\begin{aligned}
& 1-\left(1-\min _{i} a_{i}\right)^{\sum_{i=1}^{n} \mu\left(A_{(i)}\right)-\mu\left(A_{(i+1)}\right)} \\
& \leq 1-\prod_{i=1}^{n}\left(1-a_{(i)}\right)^{\mu\left(A_{(i)}\right)-\mu\left(A_{(i+1)}\right)} \\
& \leq 1-\left(1-\max _{i} a_{i}\right)^{\sum_{i=1}^{n} \mu\left(A_{(i)}\right)-\mu\left(A_{(i+1)}\right)}, \\
& 1-\left(1-\min _{i} b_{i}\right)^{\sum_{i=1}^{n} \mu\left(A_{(i)}\right)-\mu\left(A_{(i+1)}\right)} \\
& \leq 1-\prod_{i=1}^{n}\left(1-b_{(i)}\right)^{\mu\left(A_{(i)}\right)-\mu\left(A_{(i+1)}\right)} \\
& \leq 1-\left(1-\max _{i} b_{i}\right)^{\sum_{i=1}^{n} \mu\left(A_{(i)}\right)-\mu\left(A_{(i+1)}\right)}, \\
& \left(\min _{i} c_{i}\right)^{\sum_{i=1}^{n} \mu\left(A_{(i)}\right)-\mu\left(A_{(i+1)}\right)} \\
& \leq \prod_{i=1}^{n}\left(c_{(i)}\right)^{\mu\left(A_{(i)}\right)-\mu\left(A_{(i+1)}\right)} \\
& \leq\left(\max _{i} c_{i}\right)^{\sum_{i=1}^{n} \mu\left(A_{(i)}\right)-\mu\left(A_{(i+1)}\right)},
\end{aligned}
$$




$$
\begin{aligned}
& \left(\min _{i} d_{i}\right)^{\sum_{i=1}^{n} \mu\left(A_{(i)}\right)-\mu\left(A_{(i+1)}\right)} \\
& \leq \prod_{i=1}^{n}\left(d_{(i)}\right)^{\mu\left(A_{(i)}\right)-\mu\left(A_{(i+1)}\right)} \\
& \leq\left(\max _{i} d_{i}\right)^{\sum_{i=1}^{n} \mu\left(A_{(i)}\right)-\mu\left(A_{(i+1)}\right)} .
\end{aligned}
$$

So we have

$$
\begin{gathered}
\min _{i} a_{i} \leq 1-\prod_{i=1}^{n}\left(1-a_{(i)}\right)^{\mu\left(A_{(i)}\right)-\mu\left(A_{(i+1)}\right)} \leq \max _{i} a_{i}, \\
\min _{i} b_{i} \leq 1-\prod_{i=1}^{n}\left(1-b_{(i)}\right)^{\mu\left(A_{(i)}\right)-\mu\left(A_{(i+1)}\right)} \leq \max _{i} b_{i}, \\
\min _{i} c_{i} \leq \prod_{i=1}^{n}\left(c_{(i)}\right)^{\mu\left(A_{(i)}\right)-\mu\left(A_{(i+1)}\right)} \leq \max _{i} c_{i}, \\
\min _{i} d_{i} \leq \prod_{i=1}^{n}\left(d_{(i)}\right)^{\mu\left(A_{(i)}\right)-\mu\left(A_{(i+1)}\right)} \leq \min _{i} d_{i} .
\end{gathered}
$$

According to (14) and (11), we have

$$
\begin{aligned}
& \left(\left[\min _{i} a_{i}, \min _{i} b_{i}\right],\left[\max _{i} c_{i}, \max _{i} d_{i}\right]\right) \\
& \quad \leq \operatorname{I-IFC}_{\mu}\left(\tilde{a}_{1}, \ldots, \tilde{a}_{n}\right) \\
& \quad \leq\left(\left[\max _{i} a_{i}, \max _{i} b_{i}\right],\left[\min _{i} c_{i}, \min _{i} d_{i}\right]\right)
\end{aligned}
$$

that is, $\widetilde{a} \leq \operatorname{I-IFC}\left(\tilde{a}_{1}, \ldots, \tilde{a}_{n}\right) \leq \tilde{a}^{+}$.

Proposition 17. Let $\tilde{a}_{i}=\left(\left[a_{i}, b_{i}\right],\left[c_{i}, d_{i}\right]\right)(i=1,2, \ldots, n)$ be a collection of interval-valued intuitionistic fuzzy values on $X$, and let $\mu$ be a fuzzy measure on $X$. If $\widetilde{s}=([a, b],[c, d])$ is an interval-valued intuitionistic fuzzy value on $X$, then

$$
I-I F C_{\mu}\left(\tilde{a}_{1}+\widetilde{s}, \ldots, \tilde{a}_{n}+\widetilde{s}\right)=I-I F C_{\mu}\left(\tilde{a}_{1}, \ldots, \widetilde{a}_{n}\right)+\widetilde{s}
$$

Proof. Since for any $i(i=1,2, \ldots, n)$,

$$
\begin{aligned}
\tilde{a}_{i}+\widetilde{s}= & \left(\left[a_{i}+a-a_{i} a, b_{i}+b-b_{i} b\right],\left[c_{i} c, d_{i} d\right]\right) \\
= & \left(\left[1-\left(1-a_{i}\right)(1-a),\right.\right. \\
& \left.\left.\quad 1-\left(1-b_{i}\right)(1-b)\right],\left[c_{i} c, d_{i} d\right]\right) .
\end{aligned}
$$

According to (14), we have

$$
\begin{aligned}
& \operatorname{I-IFC}_{\mu}\left(\widetilde{a}_{1}+\widetilde{s}, \ldots, \widetilde{a}_{n}+\widetilde{s}\right) \\
& \quad=\left(\left[1-\prod_{i=1}^{n}\left(\left(1-a_{(i)}\right)(1-a)\right)^{\mu\left(A_{(i)}\right)-\mu\left(A_{(i+1)}\right)},\right.\right.
\end{aligned}
$$

$$
\begin{aligned}
& \left.1-\prod_{i=1}^{n}\left(\left(1-b_{(i)}\right)(1-b)\right)^{\mu\left(A_{(i)}\right)-\mu\left(A_{(i+1)}\right)}\right], \\
& {\left[\prod_{i=1}^{n}\left(c_{(i)} c\right)^{\mu\left(A_{(i)}\right)-\mu\left(A_{(i+1)}\right)},\right.} \\
& \left.\left.\prod_{i=1}^{n}\left(d_{(i)} d\right)^{\mu\left(A_{(i)}\right)-\mu\left(A_{(i+1)}\right)}\right]\right) \\
& =\left(\left[1-(1-a) \prod_{i=1}^{n}\left(1-a_{(i)}\right)^{\mu\left(A_{(i)}\right)-\mu\left(A_{(i+1)}\right)},\right.\right. \\
& \left.1-(1-b) \prod_{i=1}^{n}\left(1-b_{(i)}\right)^{\mu\left(A_{(i)}\right)-\mu\left(A_{(i+1)}\right)}\right], \\
& {\left[c \prod_{i=1}^{n}\left(c_{(i)}\right)^{\mu\left(A_{(i)}\right)-\mu\left(A_{(i+1)}\right)}\right.} \\
& \left.\left.d \prod_{i=1}^{n}\left(d_{(i)}\right)^{\mu\left(A_{(i)}\right)-\mu\left(A_{(i+1)}\right)}\right]\right) .
\end{aligned}
$$

According to Definition 8, we have

$$
\begin{aligned}
& \operatorname{I}^{-I F C}\left(\tilde{a}_{1}, \ldots, \tilde{a}_{n}\right)+\widetilde{s} \\
&=\left(\left[1-(1-a) \prod_{i=1}^{n}\left(1-a_{(i)}\right)^{\mu\left(A_{(i)}\right)-\mu\left(A_{(i+1)}\right)},\right.\right. \\
&\left.1-(1-b) \prod_{i=1}^{n}\left(1-b_{(i)}\right)^{\mu\left(A_{(i)}\right)-\mu\left(A_{(i+1)}\right)}\right], \\
& {\left[c \prod_{i=1}^{n}\left(c_{(i)}\right)^{\mu\left(A_{(i)}\right)-\mu\left(A_{(i+1)}\right)},\right.} \\
&\left.\left.d \prod_{i=1}^{n}\left(d_{(i)}\right)^{\mu\left(A_{(i)}\right)-\mu\left(A_{(i+1)}\right)}\right]\right) .
\end{aligned}
$$

Thus,

$$
\operatorname{I}_{-I F C}\left(\tilde{a}_{1}+\widetilde{s}, \ldots, \tilde{a}_{n}+\widetilde{s}\right)=\operatorname{I-IFC}\left(\tilde{a}_{1}, \ldots, \tilde{a}_{n}\right)+\widetilde{s}
$$

Proposition 18. Let $\tilde{a}_{i}=\left(\left[a_{i}, b_{i}\right],\left[c_{i}, d_{i}\right]\right)(i=1,2, \ldots, n)$ be a collection of interval-valued intuitionistic fuzzy values on $X$, and let $\mu$ be a fuzzy measure on $X$. If $r>0$; then

$$
I-I F C_{\mu}\left(r \tilde{a}_{1}, \ldots, r \tilde{a}_{n}\right)=r I-I F C_{\mu}\left(\tilde{a}_{1}, \ldots, \tilde{a}_{n}\right) .
$$

Proof. According to Definition 8, for any $i(i=1,2, \ldots, n)$ and $r>0$, we have

$$
r \widetilde{a}_{i}=\left(\left[1-\left(1-a_{i}\right)^{r}, 1-\left(1-b_{i}\right)^{r}\right],\left[\left(c_{i}\right)^{r},\left(d_{i}\right)^{r}\right]\right) .
$$


Journal of Applied Mathematics

9

According to (14), we have

$$
\begin{aligned}
& \operatorname{I}^{-I F C} C_{\mu}\left(r \tilde{a}_{1}, \ldots, r \tilde{a}_{n}\right) \\
& =\left(\left[1-\prod_{i=1}^{n}\left(\left(1-a_{(i)}\right)^{r}\right)^{\mu\left(A_{(i)}\right)-\mu\left(A_{(i+1)}\right)},\right.\right. \\
& \left.1-\prod_{i=1}^{n}\left(\left(1-b_{(i)}\right)^{r}\right)^{\mu\left(A_{(i)}\right)-\mu\left(A_{(i+1)}\right)}\right], \\
& {\left[\prod_{i=1}^{n}\left(\left(c_{(i)}\right)^{r}\right)^{\left(\mu\left(A_{(i)}\right)-\mu\left(A_{(i+1)}\right)\right)},\right.} \\
& \left.\left.\prod_{i=1}^{n}\left(\left(d_{(i)}\right)^{r}\right)^{\left(\mu\left(A_{(i)}\right)-\mu\left(A_{(i+1)}\right)\right)}\right]\right) \\
& =\left(\left[1-\prod_{i=1}^{n}\left(1-a_{(i)}\right)^{r\left(\mu\left(A_{(i)}\right)-\mu\left(A_{(i+1)}\right)\right)}\right.\right. \text {, } \\
& \left.1-\prod_{i=1}^{n}\left(1-b_{(i)}\right)^{r\left(\mu\left(A_{(i)}\right)-\mu\left(A_{(i+1)}\right)\right)}\right], \\
& {\left[\prod_{i=1}^{n}\left(c_{(i)}\right)^{r\left(\mu\left(A_{(i)}\right)-\mu\left(A_{(i+1)}\right)\right)},\right.} \\
& \left.\left.\prod_{i=1}^{n}\left(d_{(i)}\right)^{r\left(\mu\left(A_{(i)}\right)-\mu\left(A_{(i+1)}\right)\right)}\right]\right) .
\end{aligned}
$$

Since

$$
\begin{gathered}
r \mathrm{IIFC}_{\mu}\left(\tilde{a}_{1}, \ldots, \tilde{a}_{n}\right) \\
=r\left(\left[1-\prod_{i=1}^{n}\left(1-a_{(i)}\right)^{\mu\left(A_{(i)}\right)-\mu\left(A_{(i+1)}\right)},\right.\right. \\
\left.1-\prod_{i=1}^{n}\left(1-b_{(i)}\right)^{\mu\left(A_{(i)}\right)-\mu\left(A_{(i+1)}\right)}\right], \\
{\left[\prod_{i=1}^{n}\left(c_{(i)}\right)^{\mu\left(A_{(i)}\right)-\mu\left(A_{(i+1)}\right)},\right.} \\
=\left(\left[\begin{array}{l}
n \\
n
\end{array} \prod_{i=1}^{n}\left(d_{(i)}\right)^{\mu\left(A_{(i)}\right)-\mu\left(A_{(i+1)}\right)}\right]\right) \\
{\left[\prod_{i=1}^{n}\left(1-a_{(i)}\right)^{\mu\left(A_{(i)}\right)-\mu\left(A_{(i+1)}\right)}\right)^{r},} \\
{\left[\left(\prod_{i=1}^{n}\left(c_{(i)}\right)^{\mu\left(A_{(i)}\right)-\mu\left(A_{(i+1)}\right)}\right)^{r},\right.} \\
\left.\left[\prod_{i=1}^{n}\left(1-b_{(i)}\right)^{\mu\left(A_{(i)}\right)-\mu\left(A_{(i+1)}\right)}\right)^{r}\right],
\end{gathered}
$$

$$
\begin{gathered}
\left.\left.\left(\prod_{i=1}^{n}\left(d_{(i)}\right)^{\mu\left(A_{(i)}\right)-\mu\left(A_{(i+1)}\right)}\right)^{r}\right]\right) \\
=\left(\left[1-\prod_{i=1}^{n}\left(1-a_{(i)}\right)^{r\left(\mu\left(A_{(i)}\right)-\mu\left(A_{(i+1)}\right)\right)},\right.\right. \\
{\left[\prod_{i=1}^{n}\left(c_{(i)}\right)^{r\left(\mu\left(A_{(i)}\right)-\mu\left(A_{(i+1)}\right)\right)},\right.} \\
\left.\prod_{i=1}^{n}\left(1-b_{(i)}\right)^{r\left(\mu\left(A_{(i)}\right)-\mu\left(A_{(i+1)}\right)\right)}\right],
\end{gathered}
$$

thus, $\mathrm{I}-\mathrm{IFC}_{\mu}\left(r \widetilde{a}_{1}, \ldots, r \widetilde{a}_{n}\right)=r \mathrm{I}-\mathrm{IFC}_{\mu}\left(\widetilde{a}_{1}, \ldots, \widetilde{a}_{n}\right)$.

According to Propositions 17 and 18, the following coollary can be derived.

Corollary 19. Let $\tilde{a}_{i}=\left(\left[a_{i}, b_{i}\right],\left[c_{i}, d_{i}\right]\right)(i=1,2, \ldots, n)$ be a collection of interval-valued intuitionistic fuzzy values on $X$, and let $\mu$ be a fuzzy measure on $X$. If $r>0$ and $\widetilde{s}=$ $([a, b],[c, d])$ is an interval-valued intuitionistic fuzzy value on $X$,

$$
I-I F C_{\mu}\left(r \tilde{a}_{1}+\widetilde{s}, \ldots, r \tilde{a}_{n}+\widetilde{s}\right)=r I-I F C_{\mu}\left(\tilde{a}_{1}, \ldots, \tilde{a}_{n}\right)+\widetilde{s} .
$$

Remark 20. From the above analysis, the interval-valued intuitionistic fuzzy Choquet integral operator has the same properties as these of the intuitionistic fuzzy Choquet integral operator [52].

Definition 21. Let $\widetilde{a}_{i}=\left(\left[a_{i}, b_{i}\right],\left[c_{i}, d_{i}\right]\right)(i=1, \ldots, n)$ be a collection of interval-valued intuitionistic fuzzy values on $X$. An interval-valued intuitionistic fuzzy ordered weighted averaging (I-IFOWA) operator of dimension $n$ is a mapping I-IFOWA: $\Omega^{n} \rightarrow \Omega$, that has an associated weight vector $w=\left(w_{1}, w_{2}, \ldots, w_{n}\right)$ such that $w_{i} \in[0,1]$ and $\sum_{i=1}^{n} w_{i}=1$, and

$$
\begin{aligned}
& \operatorname{I-IFOWA}_{w}\left(\widetilde{a}_{1}, \tilde{a}_{2}, \ldots, \widetilde{a}_{n}\right)=w_{1} \widetilde{a}_{(1)} \\
& +w_{2} \widetilde{a}_{(2)}+\cdots+w_{n} \widetilde{a}_{(n)},
\end{aligned}
$$

where the subscript $(\cdot)$ indicates a permutation such that $\widetilde{a}_{(1)} \leq \cdots \leq \widetilde{a}_{(n)}$. Furthermore,

$$
\begin{gathered}
\operatorname{I-IFOWA}_{w}\left(\tilde{a}_{1}, \ldots, \tilde{a}_{n}\right) \\
=\left(\left[1-\prod_{i=1}^{n}\left(1-a_{(i)}\right)^{w_{i}}, 1-\prod_{i=1}^{n}\left(1-b_{(i)}\right)^{w_{i}}\right],\right. \\
\left.\left[\prod_{i=1}^{n}\left(c_{(i)}\right)^{w_{i}}, \prod_{i=1}^{n}\left(d_{(i)}\right)^{w_{i}}\right]\right) .
\end{gathered}
$$


Theorem 22. Let $\tilde{a}_{i}=\left(\left[a_{i}, b_{i}\right],\left[c_{i}, d_{i}\right]\right)(i=1,2, \ldots, n)$ be a collection of interval-valued intuitionistic fuzzy values on $X$, and let $\mu$ be a fuzzy measure on X. Any interval-valued intuitionistic fuzzy ordered weighted averaging operator with the weighted vector $w=\left(w_{1}, \ldots, w_{n}\right)$ is a special intervalvalued intuitionistic fuzzy Choquet integral operator, whose fuzzy measure $\mu$ is defined by $\mu(S)=\sum_{i=n-s+1}^{n} w_{i}(S \subseteq X, S \neq \emptyset)$ where $n, s$ is denoted the cardinality of $S, X$, respectively. Reciprocally, any commutative interval-valued intuitionistic fuzzy Choquet integral operator is such that $\mu(S)$ depends only on $s$, where $s$ denotes the cardinality of $S(S \subseteq X)$ and coincides with an interval-valued intuitionistic fuzzy ordered weighted averaging operator, whose weights are $w_{n-s}=\mu(S \cup i)-\mu(S)$, $i \in X, S \subseteq X \backslash i$.

The proof of Theorem 22 is similar to that of Proposition 12 in [52].

Remark 23. The analysis above shows that the relationship between the I-IFC operator and the I-IFOWA operator is similar to that between Choquet integral and OWA operator. Furthermore, If $\mu$ is an additive fuzzy measure on $X$ such that $\mu(A)=\sum_{i \in A} w_{i}(A \subseteq X)$, the interval-valued intuitionistic fuzzy Choquet operator is reduced to the interval-valued intuitionistic fuzzy weighted averaging operator [46]. This means that the interval-valued intuitionistic fuzzy Choquet integral operator generalizes both the interval-valued intuitionistic fuzzy ordered weighted averaging and the intervalvalued intuitionistic fuzzy weighted averaging operators. There are interactive or interdependent characteristics among decision making criteria or subjective preferences of individual decision makers; the proposed operator not only can overcome the limitation of these traditional intervalvalued intuitionistic fuzzy aggregation operators. But also can be used for better modeling the uncertain and imprecise information in the decision making process.

\section{Shapley Weights}

In multi-criteria decision making problems, weighting coefficients (weight $w_{i}$ ) are introduced to express the relative importance of different criteria. Each criterion is endowed with a specific importance weight. Generally, it is seen that weighting methods are categorized into two categories: subjective methods and objective methods [54]. Subjective methods determine weights solely based on the preference or judgments of decision makers. However, objective methods utilize mathematical models, automatically without considering the decision makers' preferences.

The VIKOR method was developed as a multi-criteria decision making method to solve a discrete decision problem with conflicting criteria [9]. For conflicting criteria, the overall importance of a criterion $i \in N$ ( $N$ denotes a criteria set) is not solely determined by itself $i$ but also by all other criteria $T, i \notin T$. Suppose that $w(i)$ denotes the importance degree of $i . w(i)=0$ suggests that the element is unimportant. For conflicting criteria, however, it may happen that for many subsets $T \subseteq N, w(T \cup i)$ is much greater than $w(T)$, suggesting that $i$ is actually an important criterion in the decision making process. So for decision making problems with conflicting criteria, we consider not only subjective weight of criteria but also objective weight. The fuzzy measure defined as above is capable of modeling this situation, which represents a weight on not only each criterion but also each combination of criteria so that the total of all the $w_{i}(i=1,2, \ldots, n)$ does not necessarily equal to one. As a result, the conflicting phenomena between criteria can be represented.

Taking this case into consideration in 1953, Shapley [55] proposed a definition of a coefficient of importance, based on a set of reasonable axioms. The importance index or Shapley value of criterion $i$ with respect to fuzzy measure $\mu$ is defined by

$$
\phi_{i}(\mu)=\sum_{T \subseteq N \backslash i} \frac{(n-t-1) ! t !}{n !}[\mu(T \cup i)-\mu(T)],
$$

where $n$ and $t$ denote the cardinality of set $N$, and $T$, respectively. Further, according to (3), for $\lambda$-fuzzy measure $g$, the importance index or Shapley value $\phi_{i}(g)$ of criterion $i$ can be expressed by

$$
\phi_{i}(g)=\sum_{T \subseteq N \backslash i} \frac{(n-t-1) ! t !}{n !} g(i) \prod_{j \in T}[1+\lambda g(j)],
$$

where fuzzy density, $g(i)$, is a subjective assessment or preference of decision maker to criterion $i$, which can be seen as a subjective weight of criterion $i$.

A basic property of the Shapley value is

$$
\sum_{i=1}^{n} \phi_{i}(\mu)=1
$$

which mean that $\left\{\phi_{i}(\mu)\right\}_{i \in N}$ is a weight vector. When $\mu$ is additive, we clearly have

$$
\phi_{i}(\mu)=\mu(i)=w_{i}, \quad i \in N .
$$

In this case decision maker's subjective preference is only taken into account in the weighting coefficients.

If $\mu$ is nonadditive then some criteria are dependent and (42) generally does not hold anymore. This shows that it is sensible to search for a coefficient of overall importance for each criterion, where the importance index or Shapley value takes into account both decision makers' subjective preference and the objective weighting of the criteria which is calculated by (40). Thus, the importance index or value of criterion is capable of being deployed as a weighting calculation method. Shapley weight provides a subjective and objective assessment of criteria in the decision making process.

\section{Extended VIKOR Method with Interval- Valued Intuitionistic Fuzzy Information}

In this section, we extend the VIKOR method to solve multicriteria group decision making problems in which all preference information provided by decision makers is expressed 
as interval-valued intuitionistic fuzzy values, and the interaction phenomena among the preference of individual decision makers and conflicting criteria are taken into account. For multi-criteria group decision making problem, let $E=$ $\left(e_{1}, e_{2}, \ldots, e_{r}\right)$ be the set of $r$ decision makers, $A=\left(a_{1}, a_{2}\right.$, $\left.\ldots, a_{m}\right)$ the set of $m$ alternatives, and $C=\left(c_{1}, c_{2}, \ldots, c_{n}\right)$ the set of $n$ criteria.

To adequately model the multi-criteria group decision making problem as above, first, the proposed interval-valued intuitionistic fuzzy Choquet integral operator is used for effectively aggregating all the opinions of individual decision makers into a collective opinion under an interval-valued intuitionistic fuzzy group decision making environment. Further, based on the VIKOR method, an interval-valued intuitionistic fuzzy VIKOR method has been developed to provide a rational, systematic decision making process by which one discovers the best solution and a compromise solution that can be used to resolve a fuzzy multi-criteria decision making problem. The extended VIKOR decision procedure of multi-criteria group decision making based on interval-valued intuitionistic fuzzy Choquet integral is summarized as follows.

Step 1. For every alternative $a_{i}(i=1,2, \ldots, m)$, each expert $e_{k}(k=1,2, \ldots, r)$ is invited to express their individual evaluations or preferences with respect to each criterion $c_{j}(j=$ $1,2, \ldots, n)$ by an interval-valued intuitionistic fuzzy value $\tilde{a}_{i j}^{k}=\left(\left[a_{\tilde{a}_{i j}^{k}}, b_{\tilde{a}_{i j}^{k}}\right],\left[c_{\tilde{a}_{i j}^{k}}, d_{\tilde{a}_{i j}^{k}}\right]\right)(i=1,2, \ldots, m ; j=1,2, \ldots, n, k=$ $1,2, \ldots, r)$, where $\left[a_{a_{i j}^{k}}, b_{a_{i j}^{k}}\right]$ indicates the uncertain degree where expert $e_{k}$ considers what the alternative $a_{i}$ should satisfy the criteria $c_{j}$ and $\left[c_{\tilde{a}_{i j}^{k}}, d_{\tilde{a}_{i j}^{k}}\right]$ indicates the uncertain degree where expert $e_{k}$ considers what the alternative $a_{i}$ should not satisfy the criteria $c_{j}$. Then we can obtain the decision making matrix as follows:

$$
D^{k}=\left(\begin{array}{c}
\widetilde{a}_{11}^{k}, \widetilde{a}_{12}^{k}, \ldots, \widetilde{a}_{1 n}^{k} \\
\vec{a}_{21}^{k}, \widetilde{a}_{22}^{k}, \ldots, \widetilde{a}_{2 n}^{k} \\
\vdots \\
\widetilde{a}_{m 1}^{k}, \widetilde{a}_{m 2}^{k}, \ldots, \widetilde{a}_{m n}^{k}
\end{array}\right) .
$$

Step 2. Since the interaction phenomena among the preference of individual decision makers are considered, the $\lambda$ fuzzy measure $g$ is used for determining the importance of each expert $e_{k}(k=1,2, \ldots, r)$, that is, the fuzzy density $g_{k}=$ $g\left(e_{k}\right)$ of each expert $e_{k}$. According to (4), the parameter $\lambda$ of expert $e_{k}$ can be calculated. Then the importance of each combination of experts can be obtained by (3).

Step 3. By (11) or Definition 7, $\widetilde{a}_{i j}^{k}$ is reordered such that $\widetilde{a}_{i j}^{(k)} \leq$ $\widetilde{a}_{i j}^{(k+1)}$. Utilize the interval-valued intuitionistic fuzzy Choquet integral operator

$$
\begin{aligned}
\tilde{a}_{i j} & =\operatorname{I}_{-I F C_{g}}\left(\tilde{a}_{i j}^{1}, \ldots, \widetilde{a}_{i j}^{r}\right) \\
& =\left(\left[1-\prod_{k=1}^{r}\left(1-a_{\tilde{a}_{i j}^{(k)}}\right)^{g\left(A_{(k)}\right)-g\left(A_{(k+1)}\right)},\right.\right.
\end{aligned}
$$

$$
\begin{gathered}
\left.1-\prod_{k=1}^{r}\left(1-b_{\widetilde{a}_{i j}^{(k)}}\right)^{g\left(A_{(k)}\right)-g\left(A_{(k+1)}\right)}\right], \\
{\left[\prod_{k=1}^{r}\left(c_{\widetilde{a}_{i j}^{(k)}}\right)^{g\left(A_{(k)}\right)-\mu\left(A_{(k+1)}\right)},\right.} \\
\left.\left.\prod_{k=1}^{r}\left(d_{\widetilde{a}_{i j}^{(k)}}\right)^{g\left(A_{(k)}\right)-\mu\left(A_{(k+1)}\right)}\right]\right)
\end{gathered}
$$

to aggregate all the interval-valued intuitionistic fuzzy decision matrices $D^{k}=\left(\widetilde{a}_{i j}^{k}\right)_{m \times n}(k=1,2, \ldots, r)$ into a collective interval-valued intuitionistic fuzzy decision matrix $D=\left(\widetilde{a}_{i j}\right)_{m \times n}$, where $\widetilde{a}_{i j}=\left(\left[a_{\widetilde{a}_{i j}}, b_{\tilde{a}_{i j}}\right],\left[c_{\tilde{a}_{i j}}, d_{\tilde{a}_{i j}}\right]\right)(i=1,2$, $\ldots, m ; j=1,2, \ldots, n), A_{(k)}=\left\{e_{(k)}, \ldots, e_{(r)}\right\}, A_{(r+1)}=\emptyset$, and $g\left(A_{(k)}\right)$ can be calculated by (3).

Step 4. Let $J_{1}$ be a collection of benefit criteria (the larger the $c_{j}$, the greater the preference) and $J_{2}$ a collection of cost criteria (the smaller the $c_{j}$, the greater the preference). The interval-valued intuitionistic fuzzy positive-ideal solution (IV-IFPIS), denoted by $\widetilde{\alpha}^{+}$, and the interval-valued intuitionistic fuzzy negative-ideal solution (IV-IFNIS), denoted by $\widetilde{\alpha}^{-}=\left(\widetilde{\alpha}_{1}^{-}, \widetilde{\alpha}_{2}^{-}, \ldots, \widetilde{\alpha}_{n}^{-}\right)$, are defined as follows:

$$
\begin{gathered}
\tilde{\alpha}^{+}=\left\{\left\langlec_{j},\left(\left[\left(\max _{i} a_{\widetilde{a}_{i j}}, \max _{i} b_{\widetilde{a}_{i j}}\right) \mid j \in J_{1},\right.\right.\right.\right. \\
\left.\left(\min _{i} a_{\widetilde{a}_{i j}}, \min _{i} b_{\widetilde{a}_{i j}}\right) \mid j \in J_{2}\right], \\
{\left[\left(\min _{i} c_{\widetilde{a}_{i j}}, \min _{i} d_{\widetilde{a}_{i j}}\right) \mid j \in J_{1},\right.} \\
\left.\left.\left.\left(\max _{i} c_{\widetilde{a}_{i j}}, \max _{i} d_{\widetilde{a}_{i j}}\right) \mid j \in J_{2}\right]\right)\right\rangle \\
\mid i=1,2, \ldots, m\} \\
=\left(\widetilde{\alpha}_{1}^{+}, \tilde{\alpha}_{2}^{+}, \ldots, \tilde{\alpha}_{n}^{+}\right),
\end{gathered}
$$

where $\widetilde{\alpha}_{j}^{+}=\left(\left[a_{\widetilde{\alpha}_{j}^{+}}, b_{\widetilde{\alpha}_{j}^{+}}\right],\left[c_{\widetilde{\alpha}_{j}^{+}}, d_{\widetilde{\alpha}_{j}^{+}}\right]\right)(j=1,2, \ldots, n)$. Consider

$$
\begin{aligned}
\tilde{\alpha}^{-}=\left\{\left\langlec_{j},\right.\right. & \left(\left[\min _{i} a_{\widetilde{a}_{i j}}, \min _{i} b_{\widetilde{a}_{i j}}\right) \mid j \in J_{1},\right. \\
& \left.\left(\max _{i} a_{\widetilde{a}_{i j}}, \max _{i} b_{\widetilde{a}_{i j}}\right) \mid j \in J_{2}\right], \\
& {\left[\left(\max _{i} c_{\widetilde{a}_{i j}}, \max _{i} d_{\widetilde{a}_{i j}}\right) \mid j \in J_{1},\right.} \\
& \left.\left.\left.\left(\min _{i} c_{\widetilde{a}_{i j}}, \min _{i} d_{\widetilde{a}_{i j}}\right) \mid j \in J_{2}\right]\right)\right\rangle
\end{aligned}
$$

$$
\begin{gathered}
\mid i=1,2, \ldots, m\} \\
=\left(\widetilde{\alpha}_{1}^{-}, \widetilde{\alpha}_{2}^{-}, \ldots, \widetilde{\alpha}_{n}^{-}\right), \\
\text {where } \widetilde{\alpha}_{j}^{-}=\left(\left[a_{\widetilde{\alpha}_{j}^{-}}, b_{\widetilde{\alpha}_{j}^{-}}\right],\left[c_{\widetilde{\alpha}_{j}^{-}}, d_{\widetilde{\alpha}_{j}^{-}}\right](j=1,2, \ldots, n) .\right.
\end{gathered}
$$


Step 5. For conflicting criteria, the $\lambda$-fuzzy measure $g$ can be used for determining the subjective assessment, that is, the importance of each criterion $c_{j}(j=1,2, \ldots, n)$, and the fuzzy density $g_{i}=g\left(c_{i}\right)$ of each criterion. According to (4), parameter $\lambda_{2}$ of criteria can be determined. Then the Shapley weight of each criterion is calculated by (39) or (40).

Step 6. Inspired by the idea of VIKOR, in this step, compute $S_{i}$ and $R_{i}$ values for $i=1,2, \ldots, m$, which symbolize the average and the worst group scores for the alternative $A_{i}$, respectively, with the relations as follows:

$$
\begin{aligned}
& S_{i} \\
& =\sum_{j=1}^{n} w_{j} \\
& \times \frac{\left(\left|a_{\widetilde{\alpha}_{j}^{+}}-a_{\widetilde{\alpha}_{i j}}\right|+\left|b_{\tilde{\alpha}_{j}^{+}}-b_{\widetilde{\alpha}_{i j}}\right|+\left|c_{\tilde{\alpha}_{j}^{+}}-c_{\widetilde{\alpha}_{i j}}\right|+\left|d_{\widetilde{\alpha}_{j}^{+}}-d_{\widetilde{\alpha}_{i j}}\right|\right)}{\left(\left|a_{\tilde{\alpha}_{j}^{+}}-a_{\tilde{\alpha}_{j}^{-}}\right|+\left|b_{\tilde{\alpha}_{j}^{+}}-b_{\tilde{\alpha}_{j}^{-}}\right|+\left|c_{\tilde{\alpha}_{j}^{+}}-c_{\tilde{\alpha}_{j}^{-}}\right|+\left|d_{\widetilde{\alpha}_{j}^{+}}-d_{\widetilde{\alpha}_{j}^{-}}\right|\right)},
\end{aligned}
$$

where $S_{i}$ is interpreted as "concordance" and can provide decision makers with information about the maximum group "utility" or "majority."

\section{Consider}

$$
\begin{aligned}
& R_{i} \\
& =\max _{j}\left(w_{j} \frac{\left(\left|a_{\tilde{\alpha}_{j}^{+}}-a_{\widetilde{a}_{i j}}\right|+\left|b_{\tilde{\alpha}_{j}^{+}}-b_{\tilde{a}_{i j}}\right|+\left|c_{\widetilde{\alpha}_{j}^{+}}-c_{\widetilde{\alpha}_{i j}}\right|+\left|d_{\widetilde{\alpha}_{j}^{+}}-d_{\widetilde{a}_{i j}}\right|\right)}{\left(\left|a_{\widetilde{\alpha}_{j}^{+}}-a_{\widetilde{\alpha}_{j}^{-}}\right|+\left|b_{\tilde{\alpha}_{j}^{+}}-b_{\widetilde{\alpha}_{j}}\right|+\left|c_{\tilde{\alpha}_{j}^{+}}-c_{\widetilde{\alpha}_{j}^{-}}\right|+\left|d_{\widetilde{\alpha}_{j}^{+}}-d_{\widetilde{\alpha}_{j}^{-}}\right|\right)}\right),
\end{aligned}
$$

where $R_{i}$ is interpreted as "discordance" and provides decision makers with information about the minimum individual regret of the "opponent."

Step 7. Compute the index values $Q_{i}$. These index values are defined as

$$
Q_{i}= \begin{cases}\frac{R_{i}-R^{-}}{R^{+}-R^{-}}, & \text {if } S^{+}=S^{-}, \\ \frac{S_{i}-S^{-}}{S^{+}-S^{-}}, & \text {if } R^{+}=R^{-} \\ \gamma \frac{S_{i}-S^{-}}{S^{+}-S^{-}}+(1-\gamma) \frac{R_{i}-R^{-}}{R^{+}-R^{-}}, & \text {otherwise }\end{cases}
$$

where $S^{+}=\operatorname{Max} S_{i}, S^{-}=\operatorname{Min} S_{i}$, which denotes that $S^{-}$ is the maximum majority rule or maximum group utility; $R^{+}=\operatorname{Max} R_{i}, R^{-}=\operatorname{Min} R_{i}$, which denotes that $R^{-}$is the minimum individual regret of the opponent. Thus, the index $Q_{i}$ is based on the consideration of both the group utility and individual regret of the opponent. In addition $\gamma$ is introduced as a weight for the strategy of "the majority of criteria" (or "the maximum group utility"), whereas $1-\gamma$ is the weight of the individual regret. The value of $\gamma$ lies in the range of $0-1$ and these strategies can be compromised by $\gamma=0.5$.

Step 8. Rank the alternatives, sorting by the values $S, R$, and $Q$ in decreasing order. The results are three ranking lists.

Step 9. Propose as a compromise solution the alternative $\left(a^{(1)}\right)$ which is the best ranked by the measure $Q$ (minimum) if the following two conditions are satisfied.

(C1) Acceptable advantage:

$$
Q\left(a^{(2)}\right)-Q\left(a^{(1)}\right) \geq D Q
$$

where $a^{(2)}$ is the alternative with second place in the ranking list by $Q ; D Q=1 /(m-1), m$ is the number of alternatives.

(C2) Acceptable stability in decision making: the alternative $a^{(1)}$ should also be the best ranked by $S$ or/and $R$. This compromise solution is stable within the decision making process, which could be the strategy of maximum group utility (when $\gamma>0.5$ is needed), or "by consensus" $\gamma \approx 0.5$, or "with veto" $(\gamma<0.5)$. Here, $\gamma$ is the weight of decision making strategy of maximum group utility.

If one of the conditions is not satisfied, then a set of compromise solutions is proposed, which consists of:

(C3) alternatives $a^{(1)}$ and $a^{(2)}$ if only the C2 is not satisfied or

(C4) alternatives $a^{(1)}, a^{(2)}, \ldots, A^{(k)}$ if the $\mathrm{C} 1$ is not satisfied; $a^{(k)}$ is determined by the relation $Q\left(a^{(k)}\right)-Q\left(a^{(1)}\right)<D Q$ for maximum $k$ (the positions of these alternatives are "in closeness").

The best alternative, ranked by $Q$, is the one with the minimum value of $Q$. The main ranking result is the compromise ranking list of alternatives and the compromise solution with "average rate." The obtained compromise solution could be accepted by the decision makers because it provides a maximum "group utility" (represented by min $S$ ) of the "majority," and a minimum of the "individual regret" (represented by $\min R$ ) of the "opponent." The compromise solutions are the basis for negotiations, involving the decision maker's preference by criteria weights.

\section{A Numerical Example}

Assume that there is an investment company who wants to invest a sum of money in the best option. There is a panel with five possible alternatives to invest the money: $a_{1}$ is a car company; $a_{2}$ is a food company; $a_{3}$ is a computer company; $a_{4}$ is an arms company; and $a_{5}$ is a TV company. The investment company must take a decision according to the following four criteria: $c_{1}$ is the profit ability; $c_{2}$ is the growth analysis; $c_{3}$ is the social-political impact; and $c_{4}$ is the enterprise culture. The five possible alternatives $a_{i}(i=1,2,3,4,5)$ are to be evaluated using the interval-valued intuitionistic fuzzy information by three decision makers $e_{k}(k=1,2,3)$, as listed in the following matrix [49]: 


$$
\begin{array}{r}
D^{1}=\left[\begin{array}{llll}
([0.4,0.5],[0.3,0.4]) & ([0.4,0.6],[0.2,0.4]) & ([0.1,0.3],[0.5,0.6]) & ([0.3,0.4],[0.3,0.5]) \\
([0.6,0.7],[0.2,0.3]) & ([0.6,0.7],[0.2,0.3]) & ([0.4,0.7],[0.1,0.2]) & ([0.5,0.6],[0.1,0.3]) \\
([0.6,0.7],[0.1,0.2]) & ([0.5,0.6],[0.3,0.4]) & ([0.5,0.6],[0.1,0.3]) & ([0.4,0.5],[0.2,0.4]) \\
([0.3,0.4],[0.2,0.3]) & ([0.6,0.7],[0.1,0.3]) & ([0.3,0.4],[0.1,0.2]) & ([0.3,0.7],[0.1,0.2]) \\
([0.7,0.8],[0.1,0.2]) & ([0.3,0.5],[0.1,0.3]) & ([0.5,0.6],[0.2,0.3]) & ([0.3,0.4],[0.5,0.6])
\end{array}\right], \\
D^{2}=\left[\begin{array}{llll}
([0.3,0.4],[0.4,0.5]) & ([0.5,0.6],[0.1,0.3]) & ([0.4,0.5],[0.3,0.4]) & ([0.4,0.6],[0.2,0.4]) \\
([0.3,0.6],[0.3,0.4]) & ([0.4,0.7],[0.1,0.2]) & ([0.5,0.6],[0.2,0.3]) & ([0.6,0.7],[0.2,0.3]) \\
([0.6,0.8],[0.1,0.2]) & ([0.5,0.6],[0.1,0.2]) & ([0.5,0.7],[0.2,0.3]) & ([0.1,0.3],[0.5,0.6]) \\
([0.4,0.5],[0.3,0.5]) & ([0.5,0.8],[0.1,0.2]) & ([0.2,0.5],[0.3,0.4]) & ([0.4,0.7],[0.1,0.2]) \\
([0.6,0.7],[0.2,0.3]) & ([0.6,0.7],[0.1,0.2]) & ([0.5,0.7],[0.2,0.3]) & ([0.6,0.7],[0.1,0.3])
\end{array}\right], \\
D^{3}=\left[\begin{array}{llll}
([0.2,0.5],[0.3,0.4]) & ([0.4,0.5],[0.1,0.2]) & ([0.3,0.6],[0.2,0.3]) & ([0.3,0.7],[0.1,0.3]) \\
([0.2,0.7],[0.2,0.3]) & ([0.3,0.6],[0.2,0.4]) & ([0.4,0.7],[0.1,0.2]) & ([0.5,0.8],[0.1,0.2]) \\
([0.5,0.6],[0.3,0.4]) & ([0.7,0.8],[0.1,0.2]) & ([0.5,0.6],[0.2,0.3]) & ([0.4,0.5],[0.3,0.4]) \\
([0.3,0.6],[0.2,0.4]) & ([0.4,0.6],[0.2,0.3]) & ([0.1,0.4],[0.3,0.6]) & ([0.3,0.7],[0.1,0.2]) \\
([0.6,0.7],[0.1,0.3]) & ([0.5,0.6],[0.3,0.4]) & ([0.5,0.6],[0.2,0.3]) & ([0.5,0.6],[0.2,0.4])
\end{array}\right] .
\end{array}
$$

To effectively solve this problem, the proposed decision procedure as above is followed for determining the most desirable alternative as follows.

Step 1. The fuzzy density of each decision maker and its $\lambda$ parameter are determined first. Suppose that $g\left(e_{1}\right)=0.40$, $g\left(e_{2}\right)=0.40$, and $g\left(e_{3}\right)=0.40$. Parameter $\lambda$ of expert can be determined: $\lambda 1=-0.44$. According to (3), we have $g\left(e_{1}, e_{2}\right)=g\left(e_{1}, e_{3}\right)=g\left(e_{2}, e_{3}\right)=0.73, g\left(e_{1}, e_{2}, e_{3}\right)=1$.

Step 2. By (11) or Definition $21, \tilde{a}_{i j}^{k}$ is reordered such that $\tilde{a}_{i j}^{(k)} \leq \tilde{a}_{i j}^{(k+1)}$; utilize the interval-valued intuitionistic fuzzy Choquet integral operator

$$
\begin{aligned}
\tilde{a}_{i j} & =\operatorname{I-IFC}{ }_{g}\left(\tilde{a}_{i j}^{1}, \tilde{a}_{i j}^{2}, \tilde{a}_{i j}^{3}\right) \\
& =\left(\left[1-\prod_{k=1}^{3}\left(1-a_{\tilde{a}_{i j}^{(k)}}\right)^{g\left(A_{(k)}\right)-g\left(A_{(k+1)}\right)},\right.\right.
\end{aligned}
$$

$$
D=\left[\begin{array}{cc}
([0.31,0.47],[0.32,0.42]) & ([0.44,0.57],[0.12,0.28]) \\
([0.42,0.68],[0.22,0.32]) & ([0.47,0.68],[0.16,0.28]) \\
([0.58,0.72],[0.13,0.24]) & ([0.59,0.70],[0.13,0.24]) \\
([0.33,0.51],[0.22,0.39]) & ([0.51,0.72],[0.12,0.26]) \\
([0.64,0.74],[0.12,0.26]) & ([0.50,0.62],[0.14,0.28])
\end{array}\right.
$$

Step 3. Since $([1,1],[0,0])$ and $([0,0],[1,1])$ are the largest and smallest interval-valued intuitionistic fuzzy values, respectively, for benefit criteria $c_{1}, c_{2}, c_{3}, c_{4}$, IV-IFPIS $\tilde{\alpha}^{+}$and IV-IFNIS $\widetilde{\alpha}^{-}$can be simply denoted as follows:

$$
\begin{array}{r}
\tilde{\alpha}^{+}=(([1,1],[0,0]),([1,1],[0,0]), \\
([1,1],[0,0]),([1,1],[0,0])), \\
\tilde{\alpha}^{-}=(([0,0],[1,1]),([0,0],[1,1]), \\
([0,0],[1,1]),([0,0],[1,1])) .
\end{array}
$$

$$
\begin{gathered}
\left.1-\prod_{k=1}^{3}\left(1-b_{\widetilde{a}_{i j}^{(k)}}\right)^{g\left(A_{(k)}\right)-g\left(A_{(k+1)}\right)}\right], \\
{\left[\prod_{k=1}^{3}\left(c_{\widetilde{a}_{i j}^{(k)}}\right)^{g\left(A_{(k)}\right)-g\left(A_{(k+1)}\right)},\right.} \\
\left.\left.\prod_{k=1}^{3}\left(d_{\widetilde{a}_{i j}^{(k)}}\right)^{g\left(A_{(k)}\right)-g\left(A_{(k+1)}\right)}\right]\right)
\end{gathered}
$$

to aggregate all decision maker's interval-valued intuitionistic fuzzy decision matrices $D^{k}=\left(\widetilde{a}_{i j}^{k}\right)_{5 \times 4}(k=1,2,3)$ into a collective interval-valued intuitionistic fuzzy decision matrix $D=\left(\widetilde{a}_{i j}\right)_{5 \times 4}$ as

$$
\left.\begin{array}{lc}
([0.29,0.50],[0.29,0.40]) & ([0.33,0.60],[0.17,0.38]) \\
([0.43,0.68],[0.12,0.22]) & ([0.54,0.72],[0.13,0.26]) \\
([0.50,0.64],[0.16,0.30]) & ([0.33,0.45],[0.29,0.45]) \\
([0.22,0.44],[0.19,0.34]) & ([0.34,0.70],[0.10,0.20]) \\
([0.50,0.64],[0.20,0.30]) & ([0.50,0.60],[0.19,0.40])
\end{array}\right]
$$

Alternatives $a_{i}(i=1,2, \ldots, 5)$ are denoted by $x_{i}=\left(\tilde{a}_{i 1}\right.$, $\left.\tilde{a}_{i 2}, \tilde{a}_{i 3}, \widetilde{a}_{i 4}\right)$ as follows:

$$
\begin{aligned}
x_{1}=(([0.31,0.47],[0.32,0.42]), \\
([0.44,0.57],[0.12,0.28]), \\
([0.29,0.50],[0.29,0.40]), \\
([0.33,0.60],[0.17,0.38])) \\
x_{2}=(([0.42,0.68],[0.22,0.32]),
\end{aligned}
$$




$$
\begin{gathered}
([0.47,0.68],[0.16,0.28]), \\
([0.43,0.68],[0.12,0.22]), \\
([0.54,0.72],[0.13,0.26])), \\
x_{3}=(([0.58,0.72],[0.13,0.24]), \\
([0.59,0.70],[0.13,0.24]), \\
([0.50,0.64],[0.16,0.30]), \\
([0.33,0.45],[0.29,0.45])), \\
x_{4}=(([0.33,0.51],[0.22,0.39]), \\
([0.51,0.72],[0.12,0.26]), \\
([0.22,0.44],[0.19,0.34]), \\
([0.34,0.70],[0.10,0.20])), \\
(([0.64,0.74],[0.12,0.26]), \\
([0.50,0.62],[0.14,0.28]), \\
([0.50,0.64],[0.20,0.30]), \\
([0.50,0.60],[0.19,0.40])) .
\end{gathered}
$$

Step 4. The fuzzy density of each criterion and its $\lambda_{2}$ parameter are calculated. Suppose that $g\left(c_{1}\right)=0.40, g\left(c_{2}\right)=0.25$, $g\left(c_{3}\right)=0.37$, and $g\left(c_{4}\right)=0.20$, according to (4); the $\lambda_{2}$ of criteria can be determined: $\lambda_{2}=-0.44$. According to (3), we have $g\left(c_{1}, c_{2}\right)=0.60, g\left(c_{1}, c_{3}\right)=0.70, g\left(c_{1}, c_{4}\right)=0.56$, $g\left(c_{2}, c_{3}\right)=0.68, g\left(c_{2}, c_{4}\right)=0.43, g\left(c_{3}, c_{4}\right)=0.54, g\left(c_{1}, c_{2}, c_{3}\right)=$ $0.88, g\left(c_{1}, c_{2}, c_{4}\right)=0.75, g\left(c_{2}, c_{3}, c_{4}\right)=0.73, g\left(c_{1}, c_{3}, c_{4}\right)=$ 0.84 , and $g\left(c_{1}, c_{2}, c_{3}, c_{4}\right)=1.0$. By means of (39), weighting coefficients of criteria are obtained as follows:

$$
w_{1}=0.32, \quad w_{2}=0.22, \quad w_{3}=0.31, \quad w_{4}=0.15 .
$$

Step 5. According to (47)-(49), the values of $R, S$, and $Q$ are calculated for all alternatives as follows.

$$
\begin{gathered}
S_{1}=0.441, \quad S_{2}=0.325, \quad S_{3}=0.321, \\
S_{4}=0.397, \quad S_{5}=0.313 \\
R_{1}=0.157, \quad R_{2}=0.116, \quad R_{3}=0.102, \\
R_{4}=0.145, \quad R_{5}=0.105, \\
Q_{1}=1, \quad Q_{2}=0.2545-0.1607 \gamma, \\
Q_{3}=0.0938 \gamma, \quad Q_{4}=0.7818-0.1295 \gamma, \\
Q_{5}=0.0545-0.0545 \gamma .
\end{gathered}
$$

Step 6. The ranking order of the alternatives by $R, S$ and $Q$ is shown in a decreasing order (see Table 1).

Step 7. From Table 1, the decision results are obtained as follows.
The best candidate (alternative) is $a_{3}$ and the ranked ordered of all candidates is $a_{3}>a_{5}>a_{2}>a_{4}>a_{1}$ if $0 \leq \gamma<0.378$; the best candidate is $a_{3}$ and $a_{5}$, the ranked order of all candidates is $a_{3} \sim a_{5}>a_{2}>a_{4}>a_{1}$ if $\gamma=0.378$; the best candidate is $a_{5}$; and the ranked order of all candidates is $a_{5}>a_{3}>a_{2}>a_{4}>a_{1}$ if $0.378<\gamma<1$; the best candidate is $a_{5}$, and the ranked ordered of all candidates is $a_{5}>a_{3} \sim a_{2}>a_{4}>a_{1}$ if $\gamma=1$.

However, for $0 \leq \gamma \leq 0.018, Q\left(a^{(2)}\right)-Q\left(a^{(1)}\right)<$ $1 /(5-1)$, the set of compromise solutions is $a_{3}$ and $a_{5}$; for $0.018<\gamma \leq 0.378, Q\left(a^{(3)}\right)-Q\left(a^{(1)}\right)<1 /(5-1)$, the set of compromise solutions is $a_{3}, a_{5}$, and $a_{2}$; for $0.378<\gamma \leq 1$, $Q\left(a^{(3)}\right)-Q\left(a^{(1)}\right)<1 /(5-1)$, the set of compromise solutions is $a_{5}, a_{3}$, and $a_{2}$.

Remark 24. In [49], Tan et al. have extended TOPSIS method to solve this decision making problems with interval-valued intuitionistic fuzzy information. Here we simply make a comparison between these two methods.

Using the extended TOPSIS [49], the ranking of this example in descending order is $a_{5}>a_{2}>a_{3}>a_{4}>a_{1}$.

Mainly, the best alternative in two methods is the same. The order of the alternatives obtained by the extended TOPSIS is litter different from that obtained by the extended VIKOR. Although the extended TOPSIS method introduces two reference points, the shortest distance from the ideal solution and the farthest distance from the negative-ideal solution, but it does not consider the relative importance of the distances from these points, which does not mean that it is always the closest to the ideal solution. In the extended VIKOR, the group majority preference to the alternative is taken into account besides individual decision-maker's preference to the alternative. So the extended VIKOR method proposes the set of compromise solutions with an advantage rate.

\section{Conclusions}

VIKOR method focused on ranking and selecting from a set of alternatives in the presence of conflicting criteria and is a helpful tool in multi-criteria decision making; the obtained compromise solution could be accepted by the decision makers because it provides a maximum group utility of the majority and a minimum of the individual regret of the opponent. The fact that social, economic, technological, and environmental factors need to be taken into consideration in decision-making makes the process more complex and need multiple decision makers to make decision together. Traditional single-person decision-making is no longer able to handle these complex problems properly. Based on an intuitionistic fuzzy Choquet integral operator, in this paper, we proposed an extension of VIKOR for effectively solving the multiple criteria group decision making problem in which the interaction phenomena among preference of the decision makers are taken into account and all the criteria values are expressed in interval-valued intuitionistic fuzzy values. Subjective and objective weights of criteria are 
TABLE 1: Decision result obtained from the extended VIKOR method.

\begin{tabular}{lccc}
\hline & $S_{i}$ & $R_{i}$ & $Q_{i}$ \\
\hline$a_{1}$ & 0.441 & 0.157 & 1 \\
$a_{2}$ & 0.325 & 0.116 & $0.2545-0.1607 \gamma$ \\
$a_{3}$ & 0.321 & 0.102 & $0.0938 \gamma$ \\
$a_{4}$ & 0.397 & 0.145 & $0.7818-0.1295 \gamma$ \\
$a_{5}$ & 0.313 & 0.105 & $0.0545-0.0545 \gamma$ \\
Ranking order & $a_{5}>a_{3}>a_{2}>a_{4}>a_{1}$ & $a_{3}>a_{5}>a_{2}>a_{4}>a_{1}$ & $a_{3}>a_{5}>a_{2}>a_{4}>a_{1}(0 \leq \gamma<0.378)$ \\
& & & $a_{3} \sim a_{5}>a_{2}>a_{4}>a_{1}(\gamma=0.378)$ \\
& & $a_{5}>a_{3}>a_{2}>a_{4}>a_{1}(0.378<\gamma<1)$ & $a_{5}>a_{3} \sim a_{2}>a_{4}>a_{1}(\gamma=1)$ \\
\hline
\end{tabular}

considered together, where subjective weights are obtained directly from the decision makers while objective weights are determined based on Shapley value. Using the proposed operator, a decision procedure is developed for solving the multi-criteria group decision making problem in an intervalvalued intuitionistic fuzzy environment. Finally we proposed a numerical example to illustrate an application of the proposed method. It shows that the proposed procedure is capable of considering the interaction phenomena between preference of experts and conflicting criteria, which makes the decision procedure more feasible and practical for solving the multi-criteria group decision-making problem.

\section{Acknowledgments}

This work was partly supported by the Funds for Creative Research Groups of China (no. 71221061), the projects of major international cooperation NSFC (no. 71210003), the National Natural Science Foundation of China (no. 71271217, 71201089), and the Program for New Century Excellent Talents in University for the Ministry of Education of China (NCET-12-0541).

\section{References}

[1] A. Goicoechea, D. R. Hansen, and L. Duckstein, Multiobjective Decision Analysis with Engineering and Business Application, John Wiley \& Sons, New York, NY, USA, 1982.

[2] P. L. Yu, "A class of solutions for group decision problems," Management Science, vol. 19, pp. 936-946, 1973.

[3] M. Zeleny, Multiple Criteria Decision Making, McGraw-Hill, New York, NY, USA, 1982.

[4] C. L. Hwang and K. Yoon, Multiple Attribute Decision Making, vol. 186 of Lecture Notes in Economics and Mathematical Systems, Springer, Berlin, Germany, 1981.

[5] S. Opricovic, Multicriteria Optimization of Civil Engineering Systems, Faculty of Civil Engineering, Belgrade, Serbia, 1998.

[6] J. P. Brans and V. Mareschal, "PROMETHEE: a new family of outranking methods in multicriteria analysis," in Operational Research, J. P. Brans, Ed., vol. 84, pp. 477-490, North-Holla, New York, NY, USA, 1984.

[7] R. Benayoun, B. Roy, and B. Sussman, ELECTRE: Une Méthode pour Guider le Choix en Présence de Points de vue Multiples, Note de Travail no. 49, Direction Scientifique: SEMA-METRA International, 1966.
[8] R. E. Steuer, Multiple Criteria Optimization: Theory, Computation, and Application, Wiley Series in Probability and Mathematical Statistics: Applied Probability and Statistics, John Wiley \& Sons, New York, NY, USA, 1986.

[9] S. Opricovic and G.-H. Tzeng, "Compromise solution by MCDM methods: a comparative analysis of VIKOR and TOPSIS," European Journal of Operational Research, vol. 156, no. 2, pp. 445-455, 2004.

[10] S. Opricovic and G.-H. Tzeng, "Extended VIKOR method in comparison with outranking methods," European Journal of Operational Research, vol. 178, no. 2, pp. 514-529, 2007.

[11] G.-H. Tzeng, C.-W. Lin, and S. Opricovic, "Multi-criteria analysis of alternative-fuel buses for public transportation," Energy Policy, vol. 33, no. 11, pp. 1373-1383, 2005.

[12] M. K. Sayadi, M. Heydari, and K. Shahanaghi, "Extension of VIKOR method for decision making problem with interval numbers," Applied Mathematical Modelling, vol. 33, no. 5, pp. 2257-2262, 2009.

[13] C.-L. Chang and C.-H. Hsu, "Multi-criteria analysis via the VIKOR method for prioritizing land-use restraint strategies in the Tseng-Wen reservoir watershed," Journal of Environmental Management, vol. 90, no. 11, pp. 3226-3230, 2009.

[14] T. Kaya and C. Kahraman, "Fuzzy multiple criteria forestry decision making based on an integrated VIKOR and AHP approach," Expert Systems with Applications, vol. 38, no. 6, pp. 7326-7333, 2011.

[15] S. Opricovic and G.-H. Tzeng, "Multicriteria planning of postearthquake sustainable reconstruction," Computer-Aided Civil and Infrastructure Engineering, vol. 17, no. 3, pp. 211-220, 2002.

[16] J. J. H. Liou, C.-Y. Tsai, R.-H. Lin, and G.-H. Tzeng, "A modified VIKOR multiple-criteria decision method for improving domestic airlines service quality," Journal of Air Transport Management, vol. 17, no. 2, pp. 57-61, 2011.

[17] J. R. San Cristóbal, "Multi-criteria decision-making in the selection of a renewable energy project in spain: the VIKOR method," Renewable Energy, vol. 36, no. 2, pp. 498-502, 2011.

[18] H. Deng and C. H. Yeh, "Simulation-based evaluation of defuzzification-based approaches to fuzzy multiattribute decision making," IEEE Transactions on Systems, Man, and Cybernetics B, vol. 36, pp. 968-977, 2006.

[19] G. J. Klir, Uncertainty and Information: Foundations of Generalized Information Theory, Wiley-Interscience, Hoboken, NJ, USA, 2006.

[20] H. Bustince, F. Herrera, and J. Montero, Fuzzy Sets and Their Extensions: Representation, Aggregation and Models, Springer, New York, NY, USA, 2007. 
[21] L. A. Zadeh, "Fuzzy sets," Information and Computation, vol. 8, pp. 338-353, 1965.

[22] K. T. Atanassov, "Intuitionistic fuzzy sets," Fuzzy Sets and Systems, vol. 20, no. 1, pp. 87-96, 1986.

[23] K. Atanassov and G. Gargov, "Interval valued intuitionistic fuzzy sets," Fuzzy Sets and Systems, vol. 31, no. 3, pp. 343-349, 1989.

[24] K. T. Atanassov, Intuitionistic Fuzzy Sets: Theory and Applications, vol. 35 of Studies in Fuzziness and Soft Computing, Physica, Heidelberg, Germany, 1999.

[25] R. R. Yager, "Fuzzy modeling for intelligent decision making under uncertainty," IEEE Transactions on Systems, Man, and Cybernetics B, vol. 30, no. 1, pp. 60-70, 2000.

[26] S. Opricovic and G.-H. Tzeng, "Fuzzy multicriteria model for postearthquake land-use planning," Natural Hazards Review, vol. 4, no. 2, pp. 59-64, 2003.

[27] G. Büyüközkan and D. Ruan, "Evaluation of software development projects using a fuzzy multi-criteria decision approach," Mathematics and Computers in Simulation, vol. 77, no. 5-6, pp. 464-475, 2008.

[28] A. Sanayei, S. F. Mousavi, and A. Yazdankhah, "Group decision making process for supplier selection with VIKOR under fuzzy environment," Expert Systems with Applications, vol. 37, no. 1, pp. 24-30, 2010.

[29] S. Opricovic, "Fuzzy VIKOR with an application to water resources planning," Expert Systems with Applications, vol. 38, no. 10, pp. 12983-12990, 2011.

[30] L. Y. Chen and T.-C. Wang, "Optimizing partners' choice in IS/IT outsourcing projects: the strategic decision of fuzzy VIKOR," International Journal of Production Economics, vol. 120, no. 1, pp. 233-242, 2009.

[31] Y. Du and P. Liu, "Extended fuzzy VIKOR method with intuitionistic trapezoidal fuzzy numbers," Information, vol. 14, no. 8, pp. 2575-2583, 2011.

[32] K. Devi, "Extension of VIKOR method in intuitionistic fuzzy environment for robot selection," Expert Systems with Applications, vol. 38, no. 11, pp. 14163-14168, 2011.

[33] J. H. Park, H. J. Cho, and Y. C. Kwun, "Extension of the VIKOR method to dynamic intuitionistic fuzzy multiple attribute decision making," Computers \& Mathematics with Applications, vol. 65, no. 4, pp. 731-744, 2013.

[34] J. H. Park, H. J. Cho, and Y. C. Kwun, "Extension of the VIKOR method for group decision making with intervalvalued intuitionistic fuzzy information," Fuzzy Optimization and Decision Making, vol. 10, no. 3, pp. 233-253, 2011.

[35] R. L. Keeney and H. Raiffa, Decisions with Multiple Objectives: Preferences and Value Tradeoffs, John Wiley \& Sons, New York, NY, USA, 1976.

[36] P. P. Wakker, Additive Representations of Preferences, Kluwer Academic Publishers, Dordrecht, The Netherlands, 1999.

[37] M. Grabisch, "Fuzzy integral in multicriteria decision making," Fuzzy Sets and Systems, vol. 69, no. 3, pp. 279-298, 1995.

[38] M. Grabisch, "The application of fuzzy integrals in multicriteria decision making," European Journal of Operational Research, vol. 89, pp. 445-456, 1996.

[39] M. Grabisch and C. Labreuche, "Fuzzy measures and integrals," in Multiple Criteria Decision Analysis State of the Art Surveys, J. Figueira, S. Greco, and M. Ehrgott, Eds., pp. 563-608, Springer, Berlin, Germany, 2005.

[40] M. Grabisch and C. Labreuche, "A decade of application of the Choquet and Sugeno integrals in multi-criteria decision aid," Annals of Operations Research, vol. 175, pp. 247-286, 2010.
[41] M. Grabisch, T. Murofushi, and M. Sugeno, Fuzzy Measures and Integrals: Theory and Applications, vol. 40 of Studies in Fuzziness and Soft Computing, Physica, Heidelberg, Germany, 2000.

[42] R. R. Yager, "On ordered weighted averaging aggregation operators in multicriteria decisionmaking," IEEE Transactions on Systems, Man and Cybernetics, vol. 18, no. 1, pp. 183-190, 1988.

[43] D. Ellsberg, "Risk, ambiguity and the Savage axioms," Quarterly Journal of Economics, vol. 75, pp. 643-669, 1961.

[44] D. Schmeidler, "Subjective probability and expected utility without additivity," Econometrica, vol. 57, no. 3, pp. 571-587, 1989.

[45] M. Sugeno, Theory offuzzy integral and its application [Doctorial dissertation], Tokyo Institute of Technology, Tokyo, Japan, 1974.

[46] Z.-S. Xu and J. Chen, "An approach to group decision making based on interval-valued intuitionistic judgment matrices," System Engineering Theory and Practice, vol. 27, no. 4, pp. 126133, 2007.

[47] Z.-S. Xu, "Methods for aggregating interval-valued intuitionistic fuzzy information and their application to decision making," Control and Decision, vol. 22, no. 2, pp. 215-219, 2007.

[48] G. W. Wei, "Some geometric aggregation functions and their application to dynamic multiple attribute decision making in the intuitionistic fuzzy setting," International Journal of Uncertainty, Fuzziness and Knowledge-Based Systems, vol. 17, no. 2, pp. 179-196, 2009.

[49] C. Tan, H. Deng, and Y. Cao, "A multi-criteria group decision making procedure using interval-valued intuitionistic fuzzy sets," Journal of Computational Information Systems, vol. 6, no. 3, pp. 855-863, 2010.

[50] C. Tan, "A multi-criteria interval-valued intuitionistic fuzzy group decision making with Choquet integral-based TOPSIS," Expert Systems with Applications, vol. 38, no. 4, pp. 3023-3033, 2011.

[51] Z. Xu, "Choquet integrals of weighted intuitionistic fuzzy information," Information Sciences, vol. 180, no. 5, pp. 726-736, 2010.

[52] C. Tan and X. Chen, "Intuitionistic fuzzy Choquet integral operator for multi-criteria decision making," Expert Systems with Applications, vol. 37, no. 1, pp. 149-157, 2010.

[53] E. P. Klement, R. Mesiar, and E. Pap, Triangular Norms, vol. 8, Kluwer Academic Publishers, Dordrecht, The Netherlands, 2000.

[54] T. C. Wang and H. D. Lee, "Developing a fuzzy TOPSIS approach based on subjective weights and objective weights," Expert System with Applications, vol. 36, no. 5, pp. 8980-8985, 2009.

[55] L. S. Shapley, "A value for $n$-person games," in Contributions to the Theory of Games, Volume II, vol. 28 of Annals of Mathematics Studies, pp. 307-317, Princeton University Press, Princeton, NJ, USA, 1953. 


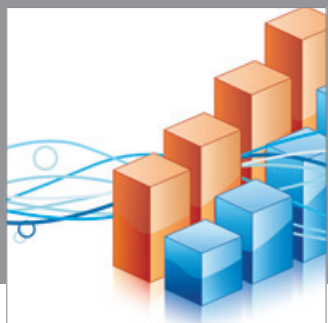

Advances in

Operations Research

mansans

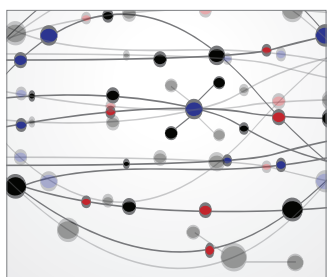

The Scientific World Journal
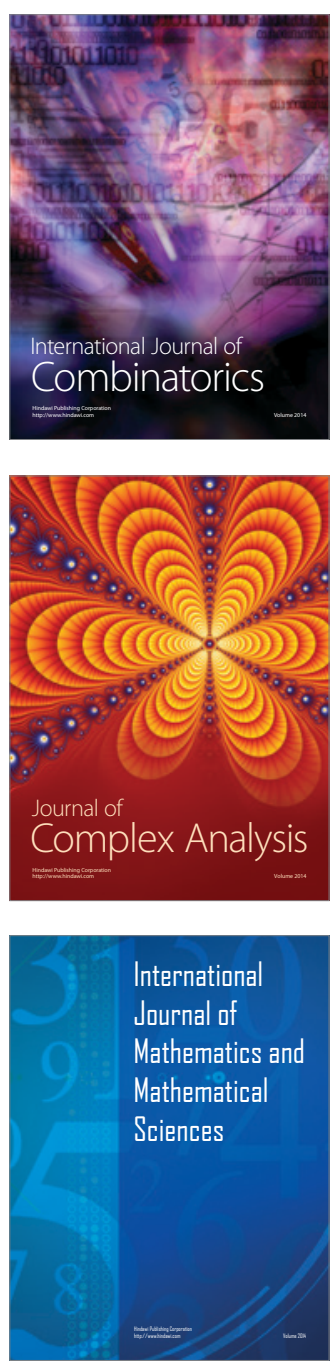
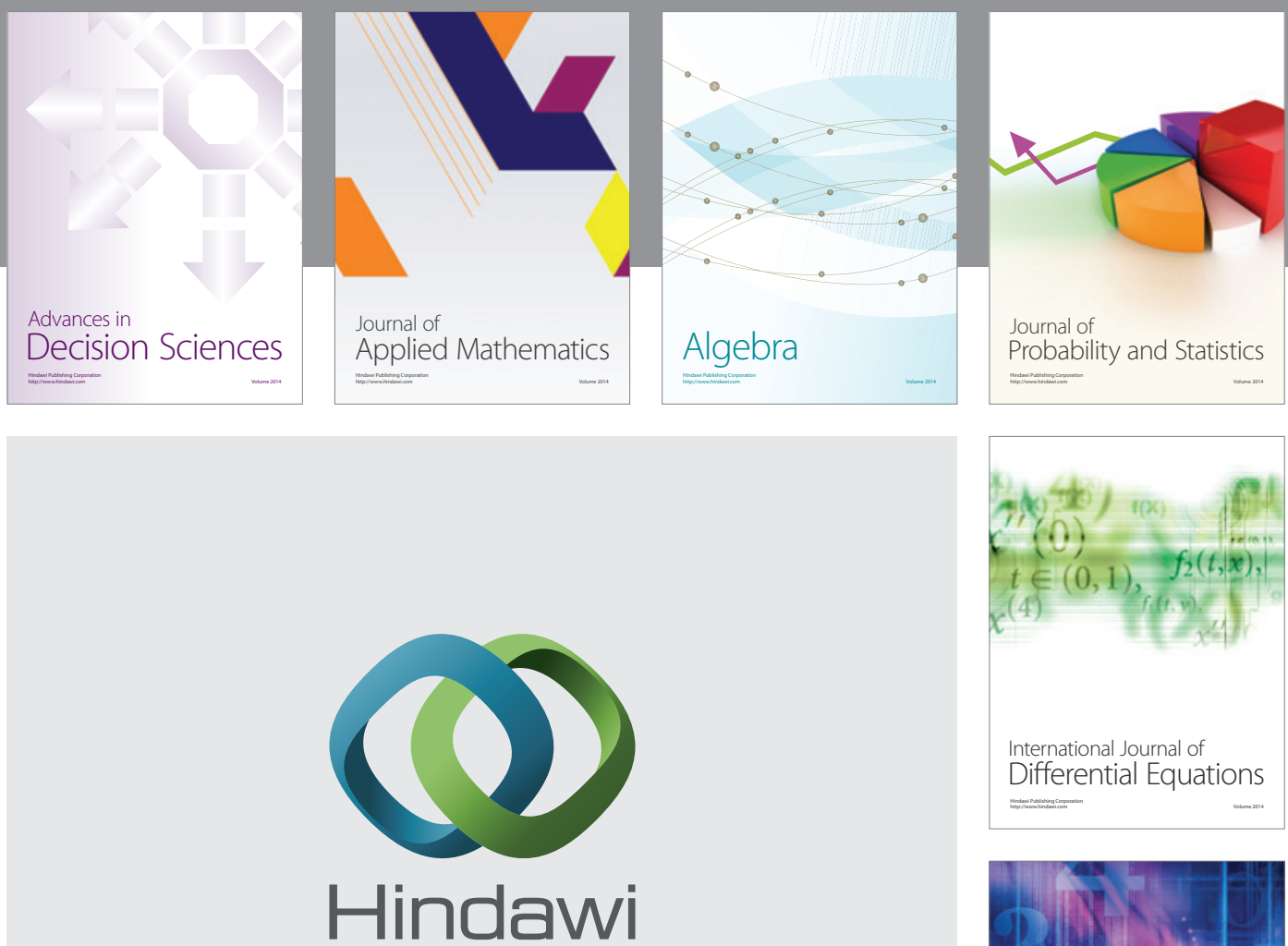

Submit your manuscripts at http://www.hindawi.com
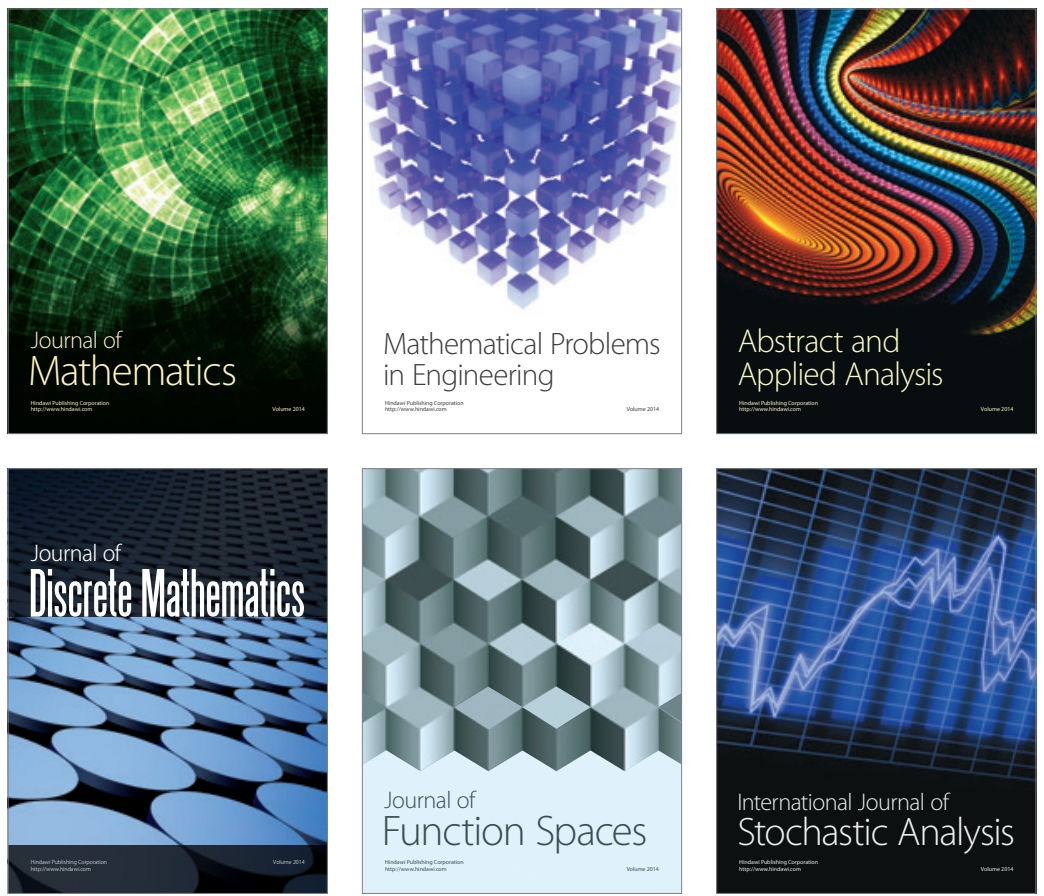

Journal of

Function Spaces

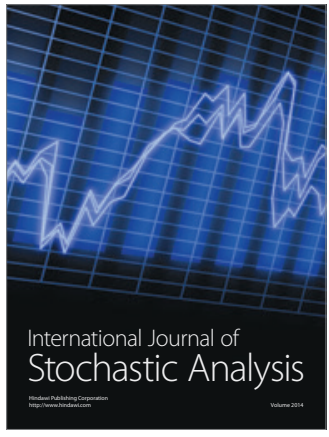

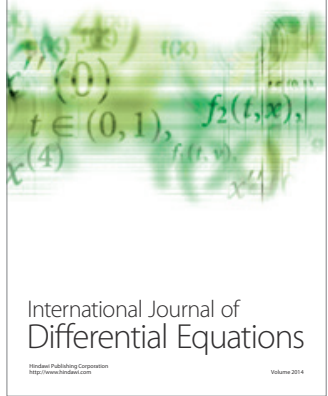
